OPEN ACCESS

Edited by:

Arun Bokde,

Trinity College Dublin, Ireland

Reviewed by:

Luiz Antonio Baccala

Universidade de São Paulo, Brazil

Yifeng Wang,

University of Electronic Science and Technology of China, China

*Correspondence:

Afra Wohlschläger

afra.wohlschlaeger@tum.de

Received: 16 July 2018 Accepted: 03 October 2018 Published: 26 October 2018

Citation:

Ries A, Chang C, Glim S, Meng $C$, Sorg $C$ and Wohlschläger $A$ (2018) Grading of Frequency Spectral Centroid Across Resting-State

Networks.

Front. Hum. Neurosci. 12:436. doi: 10.3389/fnhum.2018.00436

\section{Grading of Frequency Spectral Centroid Across Resting-State Networks}

\author{
Anja Ries, 1,2, Catie Chang ${ }^{3}$, Sarah Glim ${ }^{1,2,4}$, Chun Meng 1,2,5, Christian Sorg ${ }^{1,2,6}$ and \\ Afra Wohlschläger ${ }^{1,2 *}$
}

${ }^{1}$ Department of Neuroradiology, Technical University of Munich, Munich, Germany, ${ }^{2}$ TUM-NIC, Neuroimaging Center, Technical University of Munich, Munich, Germany, ${ }^{3}$ Advanced MRI Section, Laboratory of Functional and Molecular Imaging, National Institute of Neurological Disorders and Stroke - National Institutes of Health, Bethesda, MD, United States, ${ }^{4}$ Graduate School of Systemic Neurosciences, Ludwig-Maximilians-Universität München, Munich, Germany, ${ }^{5}$ Department of Psychiatry, University of Cambridge, Cambridge, United Kingdom, ${ }^{6}$ Department of Psychiatry, Technical University of Munich, Munich, Germany

Ongoing, slowly fluctuating brain activity is organized in resting-state networks (RSNs) of spatially coherent fluctuations. Beyond spatial coherence, RSN activity is governed in a frequency-specific manner. The more detailed architecture of frequency spectra across RSNs is, however, poorly understood. Here we propose a novel measurethe Spectral Centroid (SC)-which represents the center of gravity of the full power spectrum of RSN signal fluctuations. We examine whether spectral underpinnings of network fluctuations are distinct across RSNs. We hypothesize that spectral content differs across networks in a consistent way, thus, the aggregate representation-SCsystematically differs across RSNs. We therefore test for a significant grading (i.e., ordering) of SC across RSNs in healthy subjects. Moreover, we hypothesize that such grading is biologically significant by demonstrating its RSN-specific change through brain disease, namely major depressive disorder. Our results yield a highly organized grading of SC across RSNs in 820 healthy subjects. This ordering was largely replicated in an independent dataset of 25 healthy subjects, pointing toward the validity and consistency of found SC grading across RSNs. Furthermore, we demonstrated the biological relevance of SC grading, as the SC of the salience network-a RSN well known to be implicated in depression-was specifically increased in patients compared to healthy controls. In summary, results provide evidence for a distinct grading of spectra across RSNs, which is sensitive to major depression.

Keywords: resting-state, fMRI, resting-state networks, spectral centroid, frequency-based grading, major depressive disorder, spectral analysis, resting-state functional connectivity

\section{INTRODUCTION}

The human brain is capable of complex functions which are supported by its distinct spatiotemporal organization. Human cortical regions are arranged in a characteristic sensorimotor-to-transmodal gradient, revealing gradual variation in structural features (Huntenburg et al., 2017), macroscopic connectivity (Margulies et al., 2016) and functional specialization (Huth et al., 2016). This spatial gradient is anchored in early sensory cortical areas such as the primary visual, somatomotor, and 
auditory cortices and evolves toward higher-order transmodal areas in the parietal, temporal, and prefrontal cortex (for review see Huntenburg et al., 2018).

The human brain also exhibits a hierarchy of timescales of neural dynamics. Prior work revealed that during various task demands information integration across spatially distinct neural circuits evolves on different timescales (Hasson et al., 2008, 2015; Lerner et al., 2011; Ding et al., 2016; Baldassano et al., 2017). Primary sensory areas were found to encode instantaneous, rapidly changing information whereas transmodal association areas were shown to encode information accumulated over a longer time. Such temporal hierarchy of information integration during task relates to timescales of intrinsic (restingstate) cortical dynamics (Honey et al., 2012; Stephens et al., 2013; Murray et al., 2014). Correspondingly, early sensory areas that accumulate information over shorter timescales were found to exhibit faster resting-state fluctuations while transmodal areas that accumulate information over longer timescales showed slower resting-state fluctuations. Numerous other studies in humans and primates further support the notion of a hierarchy of resting-state timescales across individual cortical regions (Baria et al., 2011; Chaudhuri et al., 2015; Cocchi et al., 2016).

Intrinsic, slowly fluctuating brain activity is organized in so called resting-state networks (RSNs) of coherent activity (Biswal et al., 1995; Greicius et al., 2003; Fox et al., 2005; Fox and Raichle, 2007). Analysis of RSN activity and their interactions-typically by correlated resting-state fMRI signal fluctuations-constitute a powerful tool to study large-scale functional integration of the brain. Functional connectivity (FC) is a measure of signal covariance and allows for the assessment of communication between spatially distinct brain regions within (intra-FC) or between (inter-FC) networks.

Consistently with the spatial organization of resting-state dynamics of individual cortical regions, network activity at rest is also largely shaped by the temporal domain. Both intra- and inter-FC as well as the resulting network topologies-assessed via fMRI blood oxygen level dependent (BOLD) signal-were shown to be governed in a frequency specific manner (Salvador et al., 2008; Wu et al., 2008; Zuo et al., 2010; Sasai et al., 2014; Thompson and Fransson, 2015). More recently, Gollo et al. (2017) suggested that a hierarchy of timescales organizes activity between RSNs (with higher order networks showing a slower regime of activity and sensory networks faster neural dynamics) and within RSNs (with highly connected regions of a network showing slower dynamics than less interconnected, peripheral regions). Considering these findings, it is crucial to investigate brain's functional organization in a frequencyresolved fashion.

Intact temporal organization of neural dynamics determine a healthy regime of brain functioning. Deviations from this healthy regime could be reflected in malfunctioning neural processes, as is the case in several neurological disorders. Alterations in power spectra of resting-state fluctuations in individual brain regions or networks have been reported in schizophrenia (Garrity et al., 2007; Calhoun et al., 2011), bipolar disorder (Calhoun et al., 2011), and chronic pain (Malinen et al., 2010; Baliki et al., 2011). Moreover, there are numerous reports of altered FC in neurological disorders such as schizophrenia (Garrity et al., 2007; Rotarska-Jagiela et al., 2010; Dong et al., 2018), Alzheimer's disease (Greicius et al., 2004; Sorg et al., 2007; Wang et al., 2007; Sheline and Raichle, 2013; Badhwar et al., 2017), obsessive-compulsive disorder (OCD) (Zhu et al., 2016; Gürsel et al., 2018), and major depressive disorder (MDD) (Greicius et al., 2007; Wang et al., 2012; Kaiser et al., 2015; Zhong et al., 2016). Aberrant FC patterns could relate to shifts in frequency distribution of regional and network BOLD signal fluctuations. Nonetheless, spectral properties of BOLD fluctuations in many of these disorders have not yet been investigated or need to be investigated in more detail to better understand the underlying causes of the breakdown in large-scale brain communication.

As such, well-functioning interregional FC is viewed as a prerequisite of large-scale communication of neuronal assemblies, where the ongoing oscillatory processes of individual brain areas as well as of widespread networks constitute the groundwork of FC formation and should be investigated in more detail. Contrary to the initial view on fluctuating properties of RSNs-which narrows the investigatory interest to peak frequencies below $0.1 \mathrm{~Hz}$ (Cordes et al., 2001; Fox and Raichle, 2007)-it has been shown that the frequency spectrum of intrinsic fluctuations is broad and includes frequencies up to $0.25 \mathrm{~Hz}$ or even $0.75 \mathrm{~Hz}$ (Niazy et al., 2011; Boubela et al., 2013; Gohel and Biswal, 2015). Thus, RSNs represent complex processes that evolve through coherence on various temporal scales within the broad term of low-frequency fluctuations. Investigation of broadband processes is needed to preserve the richness of RSNs' operating regime, i.e., information content across the broad frequency spectrum. The more detailed architecture of frequency spectra across networks is, however, poorly understood.

Identifying key features of broadband spectra of BOLD network fluctuations-which might be also sensitive to pathological change-however, bears a challenge, given the relatively wide frequency span of meaningful resting-state BOLD fluctuations $(0.01-0.75 \mathrm{~Hz})$ and the need for frequency-resolved analysis (for example dividing the full frequency band into 3-10 sub-bands). Moreover, the vast amount of literature on aberrant resting-state BOLD activity in various diseases-often involving divergent results-points toward a vast number of regions or networks that are implicated in specific diseases. Thus, investigating group differences in spectral properties of regions/networks-of-interest within several frequency subbands would involve many statistical tests, possibly inflating false-positive results; and if strictly corrected for multiple comparisons-reduce the statistical power of the analysis. To circumvent this problem, complex measures can be summarized into meaningful aggregate measures, which may improve detection of systematic patterns and emphasize major diseaserelated alterations. These subsequently can be followed up by post hoc tests and targeted investigations yielding detailed information on the underlying changes in power spectra.

To validate the usefulness of the proposed approach, we investigate activity fluctuations within RSNs with special focus on the broad range of dominant frequencies involved in their 
time courses-assessed via an aggregate measure of BOLD spectral properties within a network. To carry out such analysis, we propose a novel measure for RSN activity analysis-the Spectral Centroid (SC). The SC of BOLD network fluctuations represents the "center of gravity" of the full power spectrum. Pictorially, it can be understood as a midpoint within the spectral density function at which the distribution is divided into two equal parts so that, figuratively speaking, if put on the tip of a pin at this midpoint-the spectral distribution would be perfectly balanced. In mathematical terms it represents a weighted mean, as explicitly given in the methods section below. In practice, the SC is a compact measure for statistical analysis. The SC has been applied in a recent study-paralelly to our investigations-to examine the slowing of neural dynamics during graded sedation with propofol (Huang et al., 2018; in this study, the SC is referred to as the "mean frequency").

We hypothesize that each RSN exhibits a distinct SC value, therefore, each RSN takes a distinct position in an ordering of all SC values-which is referred to as grading of SC across RSNs. We also aim to explore whether the grading of SCs across networks fits the prior observations of sensorimotorto-transmodal gradient of neural timescales. Furthermore, we hypothesize that a given brain disease would alter specifically such graded organization across networks. To test our hypothesis of distinct SC value in healthy subjects, we calculate the SC using high-quality and large sample size $(N=820)$ rs-fMRI data of the Human Connectome Project $\left(\mathrm{HCP}^{1}\right)$ (Smith et al., 2013; Van Essen et al., 2013). To validate the reliability and replicability of results on SC obtained from the HCP datasetalong with general concerns about replicability in neuroimaging studies (Poldrack et al., 2017)-we further examine the SC based on an independent dataset of 25 healthy subjects, acquired at our research facility. Furthermore, to examine the potential biological relevance of SC we investigate the SC grading in a major brain disease, namely MDD, in which impairments in communication (expressed through FC) between and within networks have been widely reported (for review see Kaiser et al., 2015); in particular, the major body of evidence shows abnormalities for MDD in the salience network (SN) (Seeley et al., 2007; Menon and Uddin, 2010), default mode network (DMN) (Raichle et al., 2001; Buckner et al., 2008) and central executive network (CEN) (Seeley et al., 2007; Vincent et al., 2008). Since the frequency content of a network's signal is crucial to the formation of FC with other networks, we hypothesize that aberrant FC in depression relates to possible shifts in spectral power of infra-slow BOLD fluctuations of these RSNs.

\section{MATERIALS AND METHODS}

Two separate datasets were used for this work: Dataset 1 comprises rs-fMRI images from 820 healthy subjects of the HCP, Dataset 2 comprises structural MRI and rs-fMRI data of 25 healthy controls (HCs) and 25 MDD patients-acquired at our research facility.

\footnotetext{
${ }^{1}$ https://www.humanconnectome.org
}

\section{Dataset 1: Human Connectome Project Data Specification}

We calculated the SC on a large sample size dataset of HCP from the 900 Subjects Release (S900) to present the applicability and meaningfulness of the SC as an aggregate measure of underlying oscillatory features of a network. Among other imaging modalities (task-based MEG and fMRI, structural scans), the S900 release contains rs-fMRI scans of healthy subjects, with four rs-fMRI runs collected per subject. Out of 900 subjects, the full acquisition of all four runs with $100 \%$ of collected timepoints was completed only in 820 subjects. Our analysis is based on the rs-fMRI data of those 820 subjects. Specifically, we analyze the SC on network time courses of independent components (ICs), which are freely provided for download.

Detailed information about the HCP acquisition protocols are described elsewhere (Smith et al., 2013; Uğurbil et al., 2013; Van Essen et al., 2013 ${ }^{2}$ ). In summary, participants' functional images in Dataset 1 were acquired in four runs of approximately $15 \mathrm{~min}$ each. Two runs were acquired in one session and two in another session. Participants were asked to keep their eyes open with relaxed fixation on a projected bright cross-hair on a dark background, presented in a dimmed room. Within each session, oblique axial acquisition alternated between phase encoding in right-to-left (RL) direction in one run and phase encoding in a left-to-right (LR) direction in the other run. Resting-state images were collected with the following parameters: gradient-echo EPI sequence, $\mathrm{TR}=720 \mathrm{~ms}, \mathrm{TE}=33.1 \mathrm{~ms}$, flip angle $=52^{\circ}$, field of view $=208 \times 180(\mathrm{RO} \times \mathrm{PE})$, matrix $104 \times 90(\mathrm{RO} \times \mathrm{PE})$, slice thickness $=2 \mathrm{~mm}, 72$ slices, $2.0 \mathrm{~mm}$ isotropic voxels, multiband factor $=8$, echo spacing $0.58 \mathrm{~ms}$, and bandwitdth $=2290 \mathrm{~Hz} / \mathrm{Px}$.

Within the HCP framework, fMRI data were preprocessed using the minimal preprocessing pipeline (MPP) (Glasser et al., 2013). Afterward, rs-fMRI data were denoised using FMRIB's ICA-based Xnoiseifier (FIX) (Griffanti et al., 2014; SalimiKhorshidi et al., 2014). FIX is used to auto-classify ICA components into "good" vs. "bad" ICs, and bad components are subsequently removed from the fMRI data.

Next, ICA on preprocessed and denoised (MPP + FIX) data was performed by HCP using FSL MELODIC software ${ }^{3}$. Different ICA parcellation scenarios were used with varying number of ICs $(15,25,50,100$, and 300). For each scenario, averaged time courses per IC and subjects are publicly available for download ${ }^{4}$.

\section{Data Analysis}

We based our analysis on the 50 ICs scenario. From the 50 ICs, we identified 24 RSNs in an automated way using $f s l c c-$ a FSL based function-by calculating cross-correlations (threshold $r>0.2$ ) between 50 ICs from Dataset 1 and the established RSN templates from Allen et al. (2011) (freely available for download ${ }^{5}$ ).

\footnotetext{
${ }^{2}$ https://www.humanconnectome.org/study/hcp-young-adult/document/900subjects-data-release

${ }^{3}$ https://fsl.fmrib.ox.ac.uk/fsl/fslwiki/MELODIC

${ }^{4}$ https://db.humanconnectome.org

${ }^{5}$ http://mialab.mrn.org/data/hcp/RSN_HC_unthresholded_tmaps.nii
} 


\section{Spectral centroid}

Power spectra of BOLD network fluctuations were estimated using Welch's averaged periodogram method. Welchperiodograms were calculated on time courses of each RSN per participant and run-using the built-in Matlab function pwelch, with a Hamming window of $50 \%$ overlap of successive windows-to obtain power estimates for each spectral bin. With a TR of $720 \mathrm{~ms}$ the full spectrum of accessible frequencies spans up to $0.69 \mathrm{~Hz}$ (Nyquist frequency). Results of Welch's method were restricted to the frequency range of $0.01-0.69 \mathrm{~Hz}$, omitting the very low frequencies $(<0.01 \mathrm{~Hz})$ as these are largely affected by slow drifts due to scanner hardware and cannot fully be separated from neuronal drifts. Spectral properties of the infra-slow BOLD network fluctuations were examined at the individual level by means of the SC. The SC is an integral measure obtained by evaluating the "center of gravity" of the spectrum based on frequency and magnitude information from the Welch-periodogram. The SC is calculated as the weighted mean of the frequencies present in the signal with their power as the weight, see formula below:

$$
S C=\frac{\sum_{i=1}^{N / 2+1} i \times f \times P(i)}{\sum_{i=1}^{N / 2+1} P(i)}
$$

where $f$ is the width of each spectral bin in $\mathrm{Hz}, P(i)$ is the power at the $i$ th spectral bin given in $\mathrm{Hz}$, and $N$ is the number of points in the network's BOLD time series. In Dataset 1 the respective parameters are: $f=0.0012 \mathrm{~Hz}$, and $N=1200$.

We calculated the SC of the power spectrum of BOLD network fluctuations-within the frequency range of $0.01-0.69 \mathrm{~Hz}$-for each of the 24 RSNs per participant and run. Thus, we obtain four SC values per subject and per RSN. For each RSN and subject, the mean SC of all four runs was calculated and is later referred to as the SC. In the end, we obtain one mean SC value per subject and per RSN.

In Matlab, Lilliefors tests were carried out on the set of SC values for each RSN to determine whether they follow a normal distribution ( $p<0.05$, Bonferroni-corrected for $24 \mathrm{RSNs}$ ). SC values in some of the RSNs were found to deviate from normal distribution, thus, further analysis was performed applying non-parametric tests. The Friedman's test-a non-parametric alternative to repeated measures ANOVA-was performed on the SC values with RSN as main factor. Post hoc tests were performed as Wilcoxon signed rank test for investigating significant pairwise differences in SCs between RSNs in Dataset $1(p<0.05$, Bonferroni-corrected for $24 \mathrm{RSNs}$ ).

\section{Assessment of SC dependence on RSN size}

Additionally, we investigated the relation between RSN size and its corresponding SC value. This was motivated by the possibility that, despite the high quality of provided data and careful artifact removal, spatially smaller RSNs could be differentially affected by local high-frequency motion artifacts compared to more distributed networks. The size of a given RSN was computed from group-averaged IC spatial maps provided by HCP. These spatial maps were first binarized (at a threshold of $z>10$, which corresponds to $p<0.001$ with FWE correction), next non-zero voxels were counted, and the sum of these voxels indicated the size of a network. Pearson's correlation was computed between RSN size and SC value. The results and interpretation of this analysis are presented in Supplementary Material.

\section{Dataset 2: Healthy Controls and MDD Patients \\ Subjects}

This dataset comprised anatomical and functional MRI images from 25 healthy individuals and 25 patients who suffered from recurrent MDD (see Table 1 for detailed information on demographical and clinical characteristics). Participants' data have also been used in previous studies (Manoliu et al., 2013; Meng et al., 2014). Patients with MDD were recruited from the Department of Psychiatry of the Klinikum rechts der Isar, Technische Universität München by psychiatrists. HC individuals were recruited via advertising. All participants provided informed consent in accordance with the Human Research Committee guidelines of the Klinikum rechts der Isar, Technische Universität München. All participants were examined for their medical history, underwent psychiatric interviews and psychometric assessments. Psychiatric diagnoses were based on Diagnostic and Statistical Manual of Mental Disorder-IV (DSM-IV) (American Psychiatric Association, 2000). The Structured Clinical Interview (SCID) for DSM-IV was used to assess the presence of psychiatric diagnoses (First et al., 1996). The severity of depression symptoms was measured with the Hamilton Rating Scale for Depression (HAM-D) (Hamilton, 1960), as well as the Beck Depression Inventory (BDI) (Beck et al., 1961). The global level of social, occupational, and psychological functioning was measured with the Global Assessment of Functioning Scale (GAF) (Spitzer et al., 1992). The clinical-psychometric assessment was performed by psychiatrists who have been professionally trained for SCID interviews with inter-rater reliability for diagnoses and scores of $>95 \%$. Recurrent MDD was the primary diagnosis for all patients. More detailed patient description including disease history, co-morbidity, and current medication may be found in Meng et al. (2014) as well as in the Supplementary Material.

\section{Data Acquisition and Preprocessing}

10 min of resting-state functional MRI was acquired from all participants who were instructed to keep their eyes closed, not to think of anything particular, and not to fall asleep. We obtained subjective verification that participants stayed in a state of alertness during the rs-fMRI scan by interrogating them via intercom immediately afterward. The scanning session was successfully completed in all subjects, and all subjects reportedly stayed awake during the scan.

\section{Data acquisition}

MRI data were collected on a 3-Tesla Philips Achieva scanner with an 8-channel phased-array head coil. T1-weighted structural images were acquired with a MPRAGE sequence (echo time $=4 \mathrm{~ms}$, repetition time $=9 \mathrm{~ms}$, inversion time $=100 \mathrm{~ms}$, flip 
TABLE 1 | Demographic and clinical characteristics.

\begin{tabular}{|c|c|c|c|}
\hline Measure & $\operatorname{MDD}(n=25)$ & $\mathrm{HC}(n=25)$ & MDD vs. $H^{a, b}$ \\
\hline & Mean (SD) & Mean $(S D)$ & $p$-value \\
\hline Age [years] & $48.76(14.38)$ & $44.08(14.78)$ & $>0.05^{a}$ \\
\hline Gender (m/f) & $12 / 13$ & $11 / 14$ & $>0.05^{b}$ \\
\hline Duration of MDD [years] & $16.72(10.20)$ & NA & \\
\hline Number of episodes & $5.56(2.47)$ & NA & \\
\hline Duration of current episode [weeks] & $16.56(6.62)$ & NA & \\
\hline GAF & $49.80(10.53)$ & $99.50(1.10)$ & $<0.001^{a *}$ \\
\hline HAM-D & $22.12(7.06)$ & 0 & $<0.001^{a *}$ \\
\hline $\mathrm{BDI}$ & $24.08(6.31)$ & 0 & $<0.001^{a *}$ \\
\hline
\end{tabular}

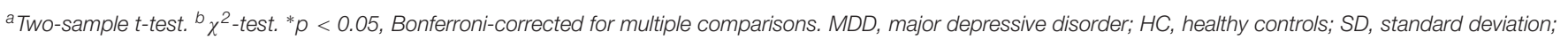
GAF, Global Assessment of Functioning Scale; HAM-D, Hamilton Depression Rating Scale; BDI, Beck Depression Inventory.

angle $=5^{\circ}$, field of view $=240 \times 240 \mathrm{~mm}$, matrix $=240 \times 240$, 170 slices, slice thickness $=1 \mathrm{~mm}$, and $0 \mathrm{~mm}$ interslice gap, voxel size $=1 \mathrm{~mm} \times 1 \mathrm{~mm} \times 1 \mathrm{~mm})$. Functional MRI data were obtained by using a gradient echo EPI sequence (echo time $=35 \mathrm{~ms}$, repetition time $=2000 \mathrm{~ms}$, flip angle $=82^{\circ}$, field of view $=220 \mathrm{~mm} \times 220 \mathrm{~mm}$, matrix $=80 \times 80,32$ slices, slice thickness $=4 \mathrm{~mm}$, and $0 \mathrm{~mm}$ interslice gap, voxel size $=2.75 \mathrm{~mm} \times 2.75 \mathrm{~mm} \times 4 \mathrm{~mm} ; 300$ volumes $)$.

\section{Preprocessing}

The first three volumes of rs-fMRI data were discarded due to magnetization effects. The remaining data were preprocessed with statistical parametric mapping software SPM $12^{6}$, including head motion correction, spatial normalization into the Montreal Neurological Institute (MNI) standard space, and spatial smoothing with a 6-mm full width at half maximum (FWHM) Gaussian filter. No slice-timing correction was performed as prior studies have shown minimal effects of slice-timing correction on fMRI data acquired at a TR of 2 s (Wu et al., 2011). Several parameters relating to head motion were investigated and compared between patients and healthy individuals to control for potential differences in motion between groups and potential bias on the results. This included the estimation of temporal signal-to-noise ratio and point-to-point head motion for each subject (Murphy et al., 2007; Van Dijk et al., 2012). Excessive head motion (cumulative motion translation or rotation $>3 \mathrm{~mm}$ or $3^{\circ}$ and mean point-to-point translation or rotation $>0.15 \mathrm{~mm}$ or $0.1^{\circ}$ ) was applied as an exclusion criterion. Point-to-point motion was defined as the absolute displacement of each brain volume compared with its previous volume. None of the participants had to be excluded. Moreover, two-sample $t$-tests showed no significant differences between groups regarding mean point-topoint translation or rotation of any direction $(p>0.1)$, as well as temporal signal-to-noise ratio $(p>0.5)$.

\section{Nuisance covariates regression}

When investigating between-group differences in SC of network BOLD fluctuations, we control for the overall percent signal change (PSC), which provides information complementary to that of SC and may help to elucidate observed differences in SC.

${ }^{6}$ http://www.fil.ion.ucl.ac.uk/spm/
PSC has been shown to differ between different RSNs (van den Heuvel et al., 2016) and might be impacted by disease (Sanacora et al., 2002; Brambilla et al., 2003; Tunnicliff and Malatynska, 2003).

Thus, throughout the analysis, we operate on two branched datasets: (1) ICA-derived time courses of RSNs-used in the examination of networks' spectral properties; (2) preprocessed whole-brain fMRI time courses-used for calculation of PSC. On that account, it is important to maintain the signal properties as consistent across the two datasets as possible. The data obtained through ICA is largely cleaned from non-neuronal variance sources, including respiratory and cardiac signals, headmovement distortions, as well as signals from white matter (WM) and cerebrospinal fluid (CSF). During ICA procedure, fluctuations of such origin are captured as ICs and are, to a great extent, separated from the remaining components characterized as RSNs.

The pre-processed whole-brain fMRI data is corrected for head movement, yet its variance may still be influenced by other sources of artifacts. To reduce the impact of these sources on the BOLD signal, regression of nuisance covariates including WM and CSF was performed. For the calculation of PSC of BOLD network fluctuations, it is highly important to exclude the variance resulting from non-neuronal factors.

Nuisance regression was performed as follows: for each participant, binarized region of interest (ROI) masks of the WM and CSF were created from T1 segmentation compartments (binarized at a threshold of 0.9). Averaged CSF and WM signals were extracted separately from individual participant data and served as covariable signals. Nuisance covariates regression was performed on the previously realigned and normalized, but not smoothed data using REST toolbox v1.8 ${ }^{7}$. Subsequently, data was spatially smoothed in SPM12 using a 6-mm FWHM Gaussian kernel.

\section{Estimation of cardiac and respiratory rates}

Physiologic Estimation by Temporal ICA (PESTICA ${ }^{8}$ ) (Beall and Lowe, 2007) was applied on raw fMRI data to detect the pulse and breathing cycles in individual subjects. In Matlab, fast Fourier

\footnotetext{
${ }^{7}$ http://restfmri.net/forum/REST

${ }^{8}$ https://www.nitrc.org/projects/pestica/
} 
transform (FFT) was used to calculate peak frequencies of cardiac and respiratory rate time courses obtained from PESTICA (with a temporal resolution of TR/slice number $=2 \mathrm{~s} / 32$ ). Concretely, a Gaussian fit in a search window was applied which corresponded to expectation values for the physiological rhythms (cardiac 55$70 \mathrm{bpm}$, beats per minute; respiratory 10-24 bpm). Visual check of fit quality was performed. Group differences in cardiac and respiratory rates were tested with two-sample $t$-tests. The tests yielded no significant difference between the groups regarding the cardiac rate $(p>0.5)$, as well as the respiratory rate $(p>0.5)$.

\section{Data Analysis}

\section{Determination of resting-state networks}

Pursuing the established approach from Allen et al. (2011), preprocessed whole-brain fMRI data were decomposed into 75 spatial ICs within a group-ICA framework (Calhoun et al., 2001) based on the infomax-algorithm and implemented in the GIFT software ${ }^{9}$. Data were concatenated and reduced by two-step principal component analysis (PCA). Next, 75 ICs were estimated by use of the Infomax algorithm, which was repeated 20 times using ICASSO $^{10}$ to reach an estimate of component reliability. The resulting set of average group ICs was then back projected into single subject space via GICA. Each back-reconstructed component is described by a spatial map of $z$-scores reflecting the FC pattern of the component across space, and an associated time course reflecting the component's BOLD activity across time. Voxels exhibiting high FC within the component have high $z$-scores and voxels that fall outside of the networks FC pattern have $z$-scores of approximately 0 . A threshold of 1 was set on the $z$-values to omit negative FC patterns within a network. The variance of component's time course after back projection is by default normalized to 1. For an automated identification of networks-of-interest, multiple spatial regression analyses on the 75 ICs were applied using established and online available templates from Allen et al. (2011) reflecting canonical RSNs. Components of highest correlation coefficient with the templates (threshold $r>0.2$ ) were selected for further analysis, resulting in 24 RSNs of interest.

\section{Percent signal change}

The spectral properties of fMRI time series would be impacted by the relative balance of signal (BOLD contrast) and noise present in the time series. The spectrum of BOLD fluctuations at rest has an approximately $1 / f^{\beta}$ distribution with frequency $f$ and power-law exponent $\beta$ (He, 2011), due in part to the low-pass character of the hemodynamic response. Therefore, assuming that low-frequency noise sources (e.g., aliased physiological noise, slow head motion, and instrumental drifts) are adequately removed, areas with smaller BOLD signal fluctuations may exhibit a distribution skewed toward higher frequencies (i.e., a shallower $1 / f$ slope) and reduced overall variance. When examining between-group differences in SC, it is important to control for the influence of PSC on the SC, as there might be systematic differences in PSC related

${ }^{9}$ http://icatb.sourceforge.net

${ }^{10}$ http://research.ics.aalto.fi/ica/icasso to pathology. Therefore, we also examined the impact of controlling for fMRI signal variance (i.e., PSC) in the analysis of SC.

To calculate the PSC, we first extracted the averaged time course corresponding to a given RSN in each subject from the preprocessed whole-brain fMRI data with and without nuisance regression. For that, binary masks for each RSN and each participant were created individually with a threshold of $z>2.32$, which corresponds to a $p$-value of 0.01 . We obtained two time courses for each network and participant (here called network_EPI_nuisance and network_EPI, respectively, for preprocessed fMRI time courses with and without nuisance regression), the PSC was calculated using the standard deviation (SD) of gray matter based signal (network_EPI_nuisance) and the mean of network_EPI signal, adhering to the established standard procedures for task-based fMRI (see e.g., Gläscher, 2009):

$$
P S C=\frac{S D(\text { network_EPI_nuisance })}{\text { mean }(\text { network_EPI })} \times 100
$$

In Matlab, Lilliefors tests were carried out on the set of PSC values for each RSN in each group to determine whether they follow a normal distribution $(p<0.05$, Bonferroni-corrected for 24 RSNs). No significant deviations from normal distribution were found. Differences in PSC between individual networks and between groups were examined via a repeated-measures ANOVA with main factors RSN and group. Post hoc tests were performed as Wilcoxon ranksum test $(p<0.05)$.

\section{Spectral density}

Power spectra of the BOLD time series from each RSN and each participant were computed in Matlab using Welch's averaged periodogram method (analogous to the procedure in Dataset 1). These spectra were subsequently used for the calculation of the SCs.

For direct comparisons of spectral power between groups, the full power spectra were then split into 10 frequency bands (freq1: 0.01-0.025; freq2: 0.025-0.05; freq3: 0.05-0.075; freq4: $0.075-0.1$; freq5: $0.1-0.125$; freq6: $0.125-0.15$; freq7: $0.15-$ 0.175; freq8: 0.175-0.2; freq9: $0.2-0.225$; and freq10: $0.225-$ $0.25 \mathrm{~Hz}$ ). The choice of frequency bands was exploratory; it constitutes a compromise between averaging for better power and sufficient spectral resolution. Mean power at each frequency band was computed and rescaled by multiplication with the corresponding PSC value. Wilcoxon ranksum tests were performed on the power values at each frequency band to test for differences between MDD and HC groups within the original as well as the rescaled power spectrum of a given network.

\section{Spectral centroid}

Spectral properties of the infra-slow BOLD network fluctuations were examined at the individual level by means of the SC (as described in eq. 1, with the following parameters: width of each spectral bin $f=0.0017 \mathrm{~Hz}$; number of points in the network's BOLD time series $N=300$ ). Next, results of Welch-periodograms were restricted to a frequency range of $0.01-0.25 \mathrm{~Hz}$, omitting the 
very low frequencies $<0.01 \mathrm{~Hz}$. From these results, we calculated the SC of the power spectrum of BOLD network fluctuations for each participant and each of the 24 RSNs. Thus, we obtain one SC value per subject and per RSN.

In Matlab, Lilliefors tests were carried out on the set of SC values for each RSN in each group to determine whether they follow a normal distribution $(p<0.05$, Bonferroni-corrected for 24 RSNs). No significant deviations from normal distribution were found. Repeated-measures ANOVA with main factors RSN and group was performed on SC values. Post hoc tests were performed as Wilcoxon ranksum tests $(p<0.05)$.

\section{Correction of spectral centroids with PSC (SCcorr)}

To rule out any differences in spectral properties related to the absolute PSC, we performed a regression analysis on the SC values and the PSC values. Separately in each group, a global regression was performed, i.e., the SC values of all RSNs in all participants within the group were pooled together and regressed against the corresponding PSC values. Regression residuals represented the "new" SC value corrected for PSC (later referred to as corrected SC; SCcorr). Repeated-measures ANOVA with RSN and group as main factors was performed on the SCcorr values.

Post hoc tests were performed as Wilcoxon ranksum tests $(p<0.05)$ to test for group differences in SCcorr of specific RSNs, and as Wilcoxon signed rank tests for investigating significant pairwise differences in SCcorr between RSNs in the control group ( $p<0.05$, Bonferroni-corrected for $24 \mathrm{RSNs})$.

\section{Assessment of SC dependence on RSN size}

As in Dataset 1, we investigated the relation between RSN size and its corresponding SC as well as SCcorr value. The size of a given RSN was computed from group-averaged binarized RSN spatial maps of HCs (obtained in an earlier step for PSC calculation). Non-zero voxels within each RSN spatial map were counted and indicated the network's size. No significant correlation between RSN size and SC or SCcorr was found (Pearson's correlation SC: $r=-0.119, p=0.58$; SCcorr: $r=-0.197$, and $p=0.36$ ).

Assesment of SC dependence on clinical scores

Pearson's correlation was calculated between SCcorr values of each RSN and measures of symptom severity $(p<0.05$, Bonferroni-corrected for 24 multiple comparisons as 24 RSNs were investigated).

\section{Network SC Correspondence Across Datasets}

Correlation analysis was run between SC values of corresponding networks obtained from Dataset 1 and networks from HCs in Dataset 2 [for a clear overview of network correspondence across study sites, i.e., Allen et al. (2011), our study, and the HCP, we refer to Supplementary Table 1]. 22 RSNs from Dataset 1 and Dataset 2 could be matched as representing the same network from Allen et al. (2011) templates. SC depends on the overall measurement length as well as on the sampling frequency, as these two parameters determine which part of the signal spectrum is accessible to analysis. E.g., with a higher sampling rate, higher frequencies can be measured which shifts
SC to slightly higher values depending on the power within these additional frequencies. Hence, SC may vary between data acquired at different facilities with different acquisition parameters. To enable the comparison of network SC values between the two datasets, results of the Welch-periodograms in Dataset 1 were restricted to the frequency range of $0.01-$ $0.25 \mathrm{~Hz}$ (as this is the frequency range accessible from Dataset 2) and-solely for comparison purposes-new SC per network was calculated on this range, so that it corresponds to the frequency range from Dataset 2. The Basal Ganglia network was excluded from the correlation analysis, as in Dataset 1 its SC exceeded two SDs. This resulted in a correlation analysis between SC values of 21 RSNs from Dataset 1 and 21 RSNs from Dataset 2.

\section{RESULTS}

\section{Dataset 1: Human Connectome Project}

The SC represents the center of gravity of the broad power spectrum of BOLD network fluctuations. We calculated the SC of 24 RSNs that corresponded to networks previously reported by Allen et al. (2011). The selected components from Dataset 1 were classified into the basal ganglia (BG; $n=1)$, auditory (AUD; $n=1$ ), sensorimotor (SM; $n=5$ ), visual (VIS; $n=5$ ), default-mode (DMN; $n=4)$, attentional (ATT; $n=5$ ), and frontal (FRONT; $n=3$ ) systems. Within the attentional systems, the $\mathrm{SN}$ and the CEN were identified. Within the $\mathrm{DMN}$, four subsystems were categorized: the anterior DMN (DMN_ant), anterior-medial DMN (DMN_antmed), posterior DMN (DMN_post) and posterior-lateral DMN (DMN_postlat). The correspondence between canonical RSN templates from Allen et al. (2011) and networks identified from Dataset 1 is described in Supplementary Table 1. Spatial maps of the 24 RSNs from Dataset 1 are shown in Figure 1. Peak activations of RSNs from Dataset 1 are summarized in Supplementary Table 2.

We assessed the SC values from ICA-derived time courses (with a spectral range of $0.01-0.69 \mathrm{~Hz}$ ) of each RSN and each participant from the HCP data. Mean SC values for each RSN are depicted in Figure 2A and summarized in Supplementary Table 2. The Friedman's test on SC values yielded a significant effect of factor RSN $\left(\chi^{2}(23)=33554.1, p<0.001\right)$. Figure 2B displays a matrix of significant pairwise differences in SC between RSNs. Columns and rows, respectively, are ordered according to SC value.

Significant grading of SC values across RSNs is observed in HCP data, implicating that SC values are characteristic for given networks, and most of the networks can be distinguished based on their SC.

\section{Dataset 2: Healthy Controls and MDD Patients \\ Resting-State Networks}

Via ICA, we identified 24 RSNs that corresponded to networks previously reported by Allen et al. (2011). The selected components were classified into the basal ganglia (BG; $n=1$ ), auditory (AUD; $n=1$ ), sensorimotor (SM; $n=6$ ), visual (VIS; $n=5)$, default-mode (DMN; $n=4)$, attentional (ATT; $n=5)$, and 


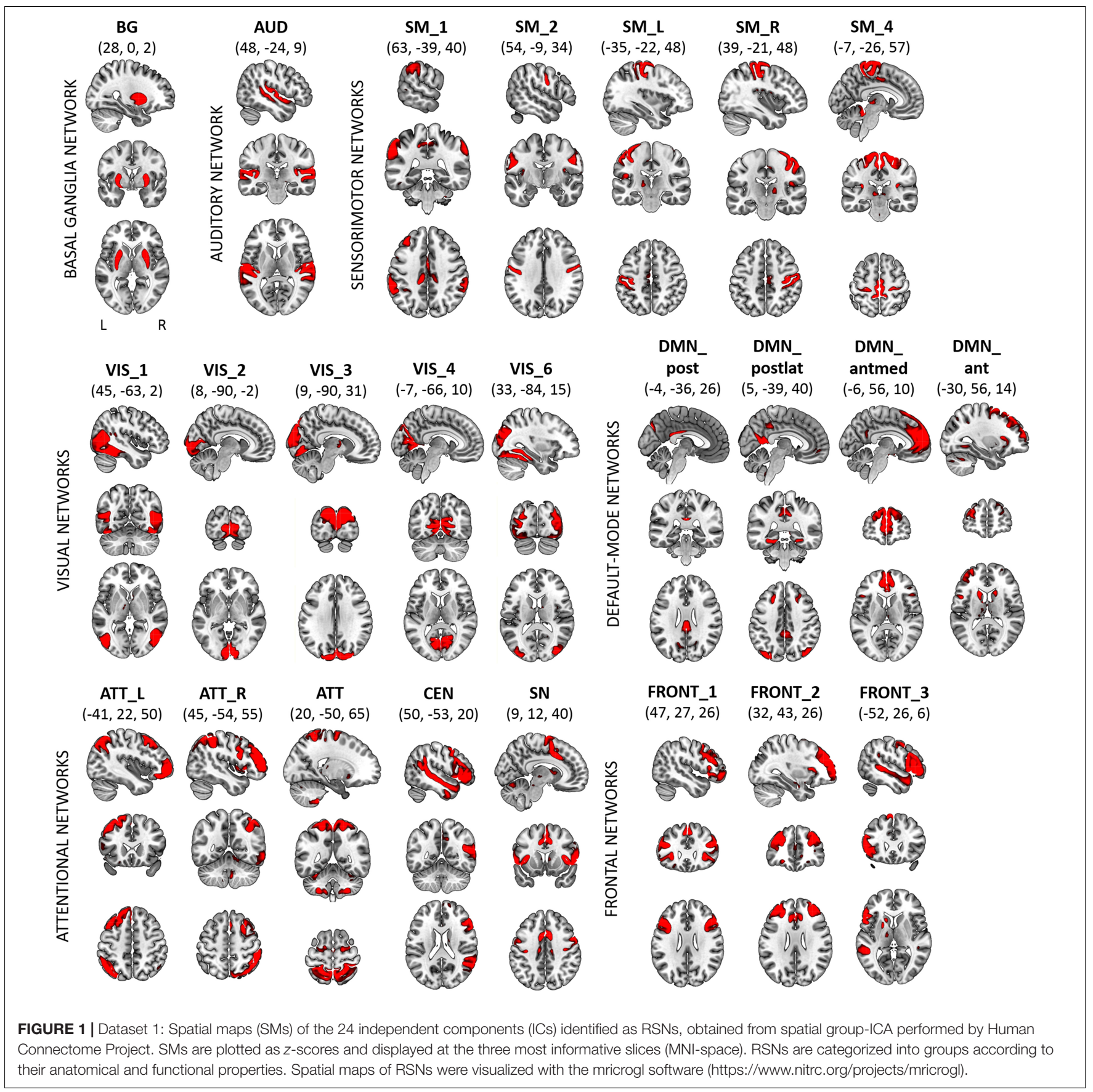

frontal (FRONT; $n=2$ ) systems. Within the attentional systems, the SN and the CEN were identified. Spatial maps of the 24 RSNs of Dataset 2 are shown in Supplementary Figure $\mathbf{1}$ and their peak activation sites are summarized in Supplementary Table 3. Correspondence between network templates from Allen et al. (2011) and names of RSNs identified from our data, as well as from HCP data is shown in Supplementary Table 1.

\section{Spectral Centroid}

We calculated the SC value for each of the 24 RSNs in HC and MDD patients (Dataset 2). The SC values of RSNs from healthy individuals in Dataset 2 and the corresponding SC values of RSNs in Dataset 1 (HCP data; obtained from power spectra restricted to $0.01-0.25 \mathrm{~Hz}$ for comparison purposes) showed a significant positive correlation $(r=0.59, p=0.004$; see Figure 3). From Figure 3 it is also clearly visible that networks of Dataset 1 exhibit smaller within-network variability in SC values (as measured with the standard error of the mean) than networks of HCs from Dataset 2.

Repeated-measures ANOVA on the SC values of Dataset 2, assessed from the spectral range of $0.01-0.25 \mathrm{~Hz}$, yielded a significant main effect of factor $\operatorname{RSN}(F(1,23)=20.64, p<0.001)$, 
Dataset 1: Human Connectome Project
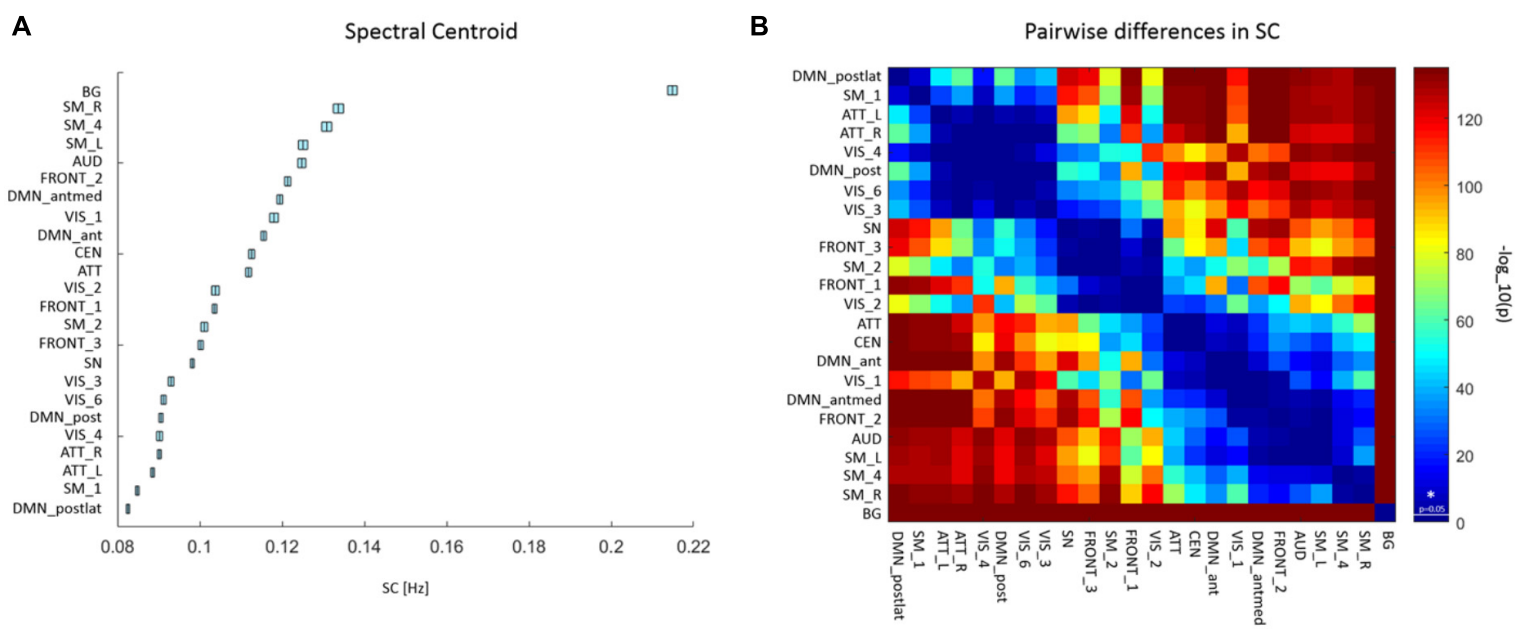

FIGURE 2 | Dataset 1: (A) Mean \pm SEM spectral centroid (SC) values of each RSN from Human Connectome Project (HCP) data of 820 healthy subjects. RSNs are ordered according to increasing SC magnitude. SCs were calculated based on power spectra of BOLD network fluctuations within the frequency range of $0.01-0.69 \mathrm{~Hz}$. (B) Matrix representing $p$-values of pairwise differences in the SC between individual RSNs. The colors indicate the significance, where red stands for lower $p$-value and higher significance, and blue for higher $p$-value thus lower significance. $P$-values were scaled with a -log10(p) transform, the colorbar value of 1.3 corresponds to $p=0.05$ (as indicated by a vertical line across the colorbar and an asterisk which represent significance threshold).

and a significant interaction between factors RSN and group $(F(1,23)=1.58, p<0.05)$. What drives the interaction can be deduced from Figure 4A, i.e., the bars representing SC values in separate groups are apart for SN and VIS_2. A post hoc Wilcoxon ranksum test yielded significant group differences in the SC of the SN ( $p<0.05$; HC mean \pm std: $0.079 \pm 0.031 \mathrm{~Hz}, \mathrm{MDD}$ mean \pm std: $0.095 \pm 0.024 \mathrm{~Hz}$ ), and a trend toward significance in one of the visual networks (VIS_2; $p=0.06$; HC mean \pm std: $0.075 \pm 0.028 \mathrm{~Hz}, \mathrm{MDD}$ mean \pm std: $0.061 \pm 0.027 \mathrm{~Hz}$ ). Mean SC values for each RSN in both groups are depicted in Figure 4A and summarized in Supplementary Table 4. To control for a potential interaction between power spectra and total power, PSC was calculated and regressed out from the SC values.

\section{Percent Signal Change}

On PSC values, repeated-measures ANOVA yielded a significant main effect of factor $\operatorname{RSN}(F(1,23)=11.5, p<0.001)$ as well as a significant interaction between factors RSN and group $(F(1,23)=2.74, p<0.001)$. Wilcoxon ranksum test was performed as part of post hoc analysis to define which RSNs show significant alterations in PSC between the groups. This analysis yielded a significant group difference in PSC in one of the visual networks (VIS_2; $p<0.05$ ). Within this network, increased PSC was observed in MDD patients (mean \pm std: $0.471 \pm 0.162 \%$ ) compared to controls (mean \pm std: $0.374 \pm 0.119 \%$ ). The PSC in another visual network (VIS_4) showed a trend toward significance $(p=0.065)$, with higher values in MDD patients (mean \pm std: $0.447 \pm 0.198 \%$ ) than in controls (mean \pm std: $0,327 \pm 0.078 \%)$. Mean PSC values for each RSN in both groups are depicted in Figure 4C and summarized in Supplementary Table 4.

\section{Corrected Spectral Centroid}

The original SC values were corrected for differences in PSC at the level of each RSN and participant. A repeated-measures ANOVA was performed on the corrected SC (SCcorr) values with RSN and group as main factor. This analysis yielded a significant main effect of factor $\operatorname{RSN}(F(1,23)=17.84, p<0.001)$. No significant interaction between factors RSN and group was observed $(F(1,23)=1.14, p>0.05)$. The averaged SCcorr values for each RSN, separately for controls and MDD patients, are displayed in Figure $4 \mathrm{~B}$ and summarized in Supplementary Table 4.

Figure 4D displays a matrix of significant pairwise differences in SCcorr between RSNs in HCs. Columns and rows, respectively, are ordered according to SCcorr magnitude. The results of Wilcoxon signed rank tests on the SCcorr values between each pair of RSNs in HCs showed significant pairwise differences in all networks with a minimum difference in SCcorr of 0.0001. At the given statistical power, only differences between networks from both ends of the SCcorr scale become statistically significant.

Since the analysis of SC before the correction for PSC showed significant differences in the SC of the $\mathrm{SN}$, and because we hypothesized a disturbance in the oscillatory pattern of the $\mathrm{SN}$ specifically-as it is widely implicated in depression-a post hoc analysis was performed to investigate group differences in SCcorr values of the SN. Moreover, since we observed a trend toward significance in the SC of visual network VIS_2, in the post hoc analysis we also investigated whether the SCcorr of this network significantly differs between groups.

The Wilcoxon rank-sum test revealed a significant difference in the SCcorr of the SN between groups $(p<0.05)$, where MDD patients showed higher SCcorr value (mean \pm std: $0.023 \pm 0.025)$ than controls (mean \pm std: $0.009 \pm 0.03$ ). After correction for 


\section{Correlation between SCs of corresponding RSNs: Dataset 1 \& Dataset 2}

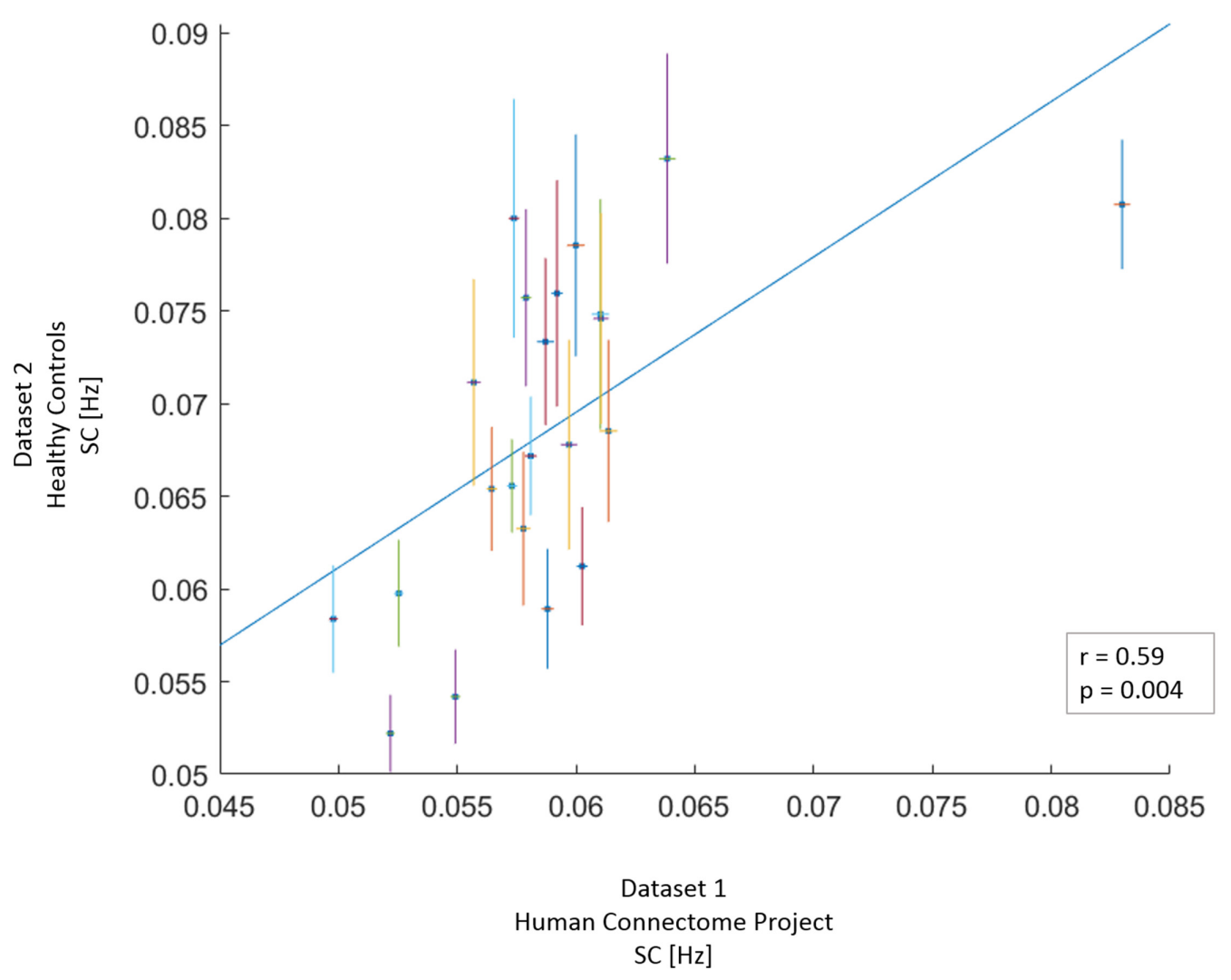

FIGURE 3 | Correlation between SC values of 21 networks obtained from Dataset 1 from signal in frequency range of $0.01-0.25 \mathrm{~Hz}$ ( $x$-axis) and the corresponding networks obtained from healthy controls in Dataset 2 ( $y$-axis). For each network, the horizontal line represents the SEM in Dataset 1 , and the vertical line represents the SEM in Dataset 2. A least-squares fit line was added to scatter plot. In the lower right box, correlation coefficient $r$ and $p$-value are presented.

PSC, there was no more significant group difference in the SCcorr of the visual network VIS_2 $(p>0.05)$. As such, the previously observed shift in SC of visual network VIS_2 could be fully attributed to differences in PSC of this network.

\section{Salience Network: Spectral Differences Between Groups}

The SC serves as an aggregate measure which is highly sensitive in detecting key changes in spectral properties of BOLD network fluctuations, assessed uniformly from the broad range of accessible frequencies-i.e., without division into frequency sub-bands. As such, the SC initially indicates network-specific spectral alterations, whereafter targeted post hoc tests examining power changes at specific frequency subbands can be performed. In that way, the drawback of multiple comparisons can be circumvented-as further detailed investigations of power changes at multiple sub-bands are restricted to networks with significantly changed SC. Thus, the SC measure is highly relevant for identifying key changes in broadband BOLD network fluctuations and for driving targeted post hoc analyses.

In Dataset 2, a significant change in SCcorr of the SN was observed in MDD patients, compared to HCs. To further investigate the causes of the shift in SCcorr value within the $\mathrm{SN}$, we examined the spectral power of SN fluctuations at each of the 10 frequency bands in both groups. The original power spectrum of the $\mathrm{SN}$, as well as the power spectrum rescaled by multiplication with PSC are shown in Figure 4E. Analysis of the original power spectrum of the $\mathrm{SN}$ yielded a significant increase in power in higher frequency bands (freq7 0.15-0.175 Hz; freq8 $0.175-0.2 \mathrm{~Hz}$; and freq10 $0.225-0.25 \mathrm{~Hz}$ ) in MDD patients compared to HCs $(p<0.05)$. There was also a tendency of decreased power in the lowest frequency band (freq1 0.01$0.025 \mathrm{~Hz})$ in MDD patients compared to HCs $(p=0.07)$. Within the rescaled power spectrum of $\mathrm{SN}$, we observed a significant decrease in power of the lowest frequency band (freq1) in MDD patients $(p<0.05)$, and a significant increase in frequency band 7 in MDD patients $(p<0.05)$. Additionally, a tendency of increased 


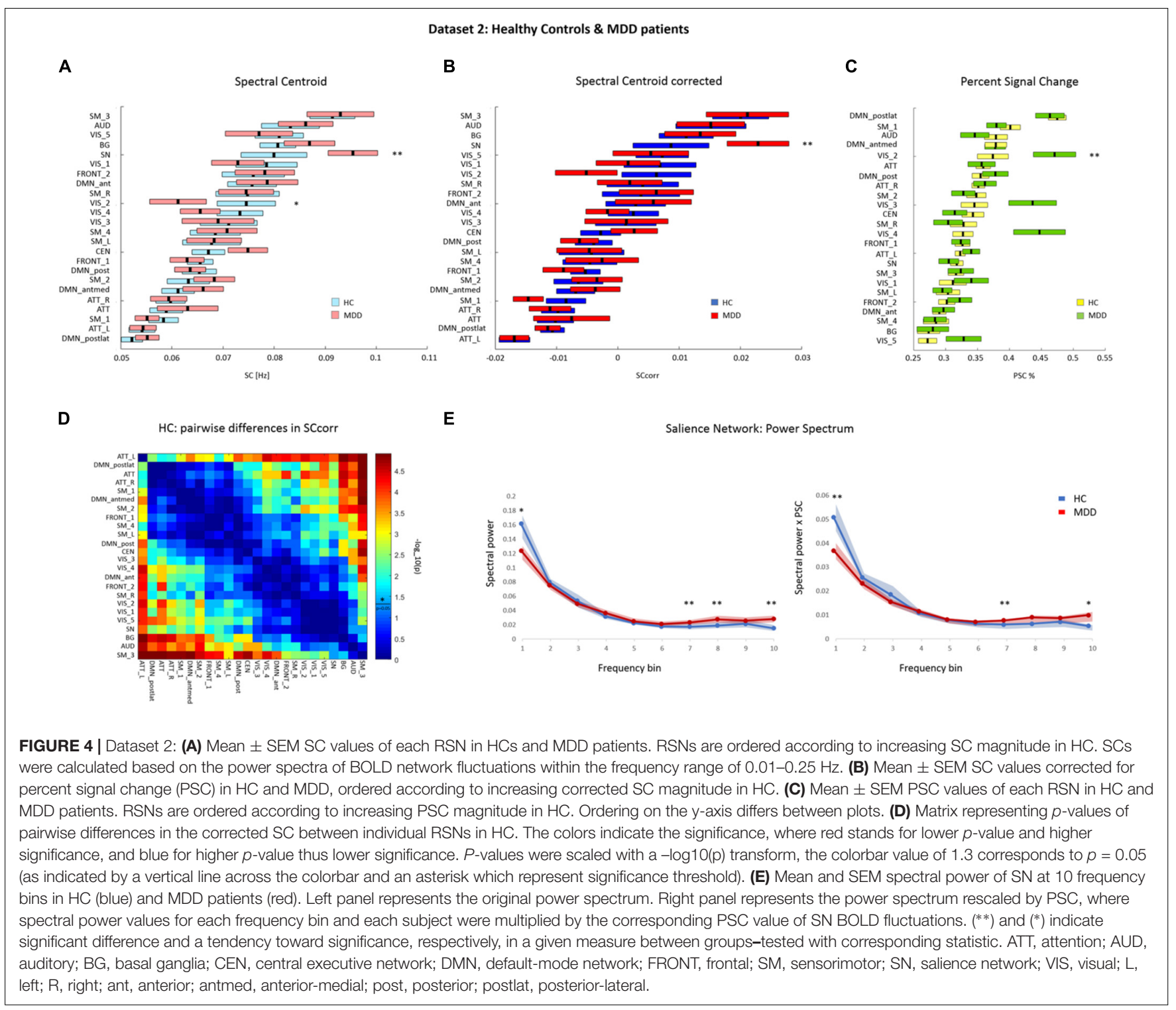

power in frequency band 10 was observed in MDD patients $(p=0.07)$. Attenuated power in lower frequency band on the one hand, and increased power in higher frequency bands on the other, result in a shift of the SC in SN toward higher frequencies.

\section{SCcorr and Symptoms Severity}

Pearson's correlation was calculated between the SCcorr values of each RSN and the symptom severity scores. We found a significant correlation between BDI scores and the SCcorr of the anterior subdivision of the DMN (DMN_ant: $r=0.62$, $p=0.001$ ) and of the posterior subdivisions of the DMN (DMN_postlat: $r=0.65, p=0.0006$; DMN_post: $r=0.58$, $p=0.002)$. There was also a trend toward significance in the correlation between BDI scores and the SCcorr of one of the visual networks (VIS_4: $r=0.53, p=0.008$ ). No significant correlation between symptom severity scores and the SCcorr of the SN was observed.
However, an indirect influence of altered SN dynamics on depression severity could be drawn based on findings from a subsequent whole-brain seed-based FC analysis of the SN (for methods and results see Supplementary Material). Reduced FC of the SN toward the anterior DMN and one of the visual networks (VIS_4)-two networks which showed significant correlation between their SCcorr and BDI-was found in MDD patients (Supplementary Figure 2). This observation and its putative consequences are further reviewed in the Discussion part.

\section{DISCUSSION}

Spectral analysis of BOLD fluctuations performed on highquality large sample dataset of Human Connectome Projectincluding 820 healthy subjects-revealed a significant grading of SC across networks. This observation could successfully be replicated through an independent dataset of 25 healthy subjects. 
Network orderings in both datasets under investigation proved to be highly correlated. Thus, the grading of SC proves to be highly reliable across two independent datasets.

The biological relevance of SC as a meaningful representative of networks' spectral properties was validated via disorder effect. In a dataset of 25 patients suffering from MDD the grading of SC was found to be altered. Specifically, the SC of the SN showed a significant shift toward higher frequencies.

Additionally, a grading of PSC across RSNs was observed in the data of 25 healthy subjects-implicating significantly distinct levels of absolute BOLD activity within specific neuronal ensembles. The grading of PSC was also affected by major depression, where significant increase in PSC of secondary visualoccipital network was found.

\section{Grading of Spectral Centroid across RSNs}

The first striking finding of our study is the occurrence of a highly organized grading of the SCs of BOLD network fluctuations at infra-slow (i.e., $<1 \mathrm{~Hz}$ ) frequencies in healthy subjects. The SC was introduced as a novel measure describing the center of gravity of the full power spectrum of restingstate BOLD network fluctuations. Our investigations on HCP dataset revealed that different RSNs involve and operate on distinct broadband frequency patterns, via which information can selectively be exchanged between targeted systems. To validate the generalizability and replicability of the finding of SC characteristic for RSNs, we repeated the analysis on an independent dataset of $25 \mathrm{HCs}$ acquired at our research facility. In line with main findings on HCP rs-fMRI data, spectral analysis on our dataset also provided a significant grading of the SCs of the BOLD network fluctuations at infra-slow frequencies. Correlation analysis between the SCs of individual RSNs from Dataset 1 and the corresponding RSNs from Dataset 2 confirms the reproducibility of SC as network property, given the different acquisition sites, parameters and analysis pipeline characteristics. The findings on HCs in Dataset 2 further highlight and support the meaningfulness of SC measure as a representative of underlying neuronal features of RSNs, by means of which a general organization-i.e., grading-of neuronal systems according to their spectral properties can be described.

Such grading is-in electrophysiology-believed to be characteristic for a highly interactive system of network oscillators (Buzsáki, 2006) and enables a degree of communication between oscillators, for only networks with overlapping oscillatory profiles are preferentially able to synchronize with each other and form FC. Respectively, any deviation from a network's healthy oscillation profile would result in a desynchronization between its own signal and the signal of networks it communicates to, thus, in a breakdown of the brain's large-scale communication structure. Likewise, in BOLD signal-which relates rather to fluctuations in broadband power of electrical oscillation-FC between any two brain areas can only arise with overlapping frequency spectra. Alterations of the spectra are therefore indicative of lack of communication.
Several studies reported that resting-state BOLD fluctuations show frequency-dependent anatomically restricted spatial structure in the human brain (Salvador et al., 2008; Wu et al., 2008; Zuo et al., 2010; Baria et al., 2011; Kalcher et al., 2014). Salvador et al. (2008) examined frequency-dependent FC profiles and found that limbic and temporal regions display highest level of oscillation coherence in high $(0.17-0.25 \mathrm{~Hz})$ and middle $(0.08-0.17 \mathrm{~Hz})$ frequency bands, while at low frequencies $(<0.08 \mathrm{~Hz})$ greatest connectivity is observed in frontal structures. Convergently, Baria et al. (2011) provided evidence of whole-brain organization of BOLD oscillatory activity within the full spectrum of frequencies available from rs-fMRI. They showed that the power of BOLD fluctuations within high-frequency band $(0.15-0.20 \mathrm{~Hz})$ is most prominent in the temporal and sub-cortical regions as well as in the insula; whereas power of BOLD oscillation in low-frequency band $(0.01-0.05 \mathrm{~Hz})$ was most accentuated in the prefrontal, occipital, and parietal lobes. Kalcher et al. (2014) showed that cortical regions exhibit highest contribution of low-frequency signals $(<0.25 \mathrm{~Hz})$, while time courses in subcortical regions as well as the insula are strongly influenced by high-frequency fluctuations $(0.25-1.4 \mathrm{~Hz})$. More recently, a hierarchical organization of timescales of intrinsic dynamics has been suggested, highlighting the sensorimotor-to-transmodal temporal gradient (Honey et al., 2012; Stephens et al., 2013; Gollo et al., 2015, 2017; Cocchi et al., 2016; for review see Huntenburg et al., 2018). According to this gradient, early sensory cortical areas such as the primary visual, somatomotor and auditory cortices operate on faster intrinsic dynamics, while the frequency content becomes dominated by lower frequencies as the gradient shifts toward higher-order transmodal areas in the parietal, temporal and prefrontal cortex. Such organization was also observed in context of large-scale brain networks (Gollo et al., 2015, 2017); early sensory RSNs showed faster dynamics than high-order RSNs; and within RSNs, highly interconnected brain regions exhibited a slower dynamic regime than less interconnected peripheral regions.

In partial accordance to the aforementioned findings, our analysis on HCP data revealed the following networks as showing highest SC values, thus, the strongest influence of high-frequency power on the network fluctuations profile: basal ganglia network (main hubs in putamen, caudate nucleus, and pallidum), early sensorimotor networks (SM_R, SM_4, SM_L; main hubs in precentral gyrus and paracentral lobule), auditory network (main hubs in superior temporal gyrus). On the other end of the power distribution, with relatively low SC values, the following networks were situated: posteriorlateral DMN (main hubs in the posterior cingulate cortex (PCC), precuneus, cuneus, angular gyrus, and IPL) and posterior DMN (main hubs in PCC, precuneus, cuneus, and IPL); sensorimotor network (SM_1, main hubs in the supramarginal gyrus and IPL); bilateral attention networks (ATT_L, ATT_R; main hubs in inferior and middle frontal gyri, IPL, and angular gyrus). Some of the visual networks (VIS_4, VIS_6, and VIS_3) were also observed at the lower end of the spectrum, however, these networks represent higher-order systems, which fall toward the end of a modality's hierarchy of processing complexity. 


\section{Dataset 1: Human Connectome Project}

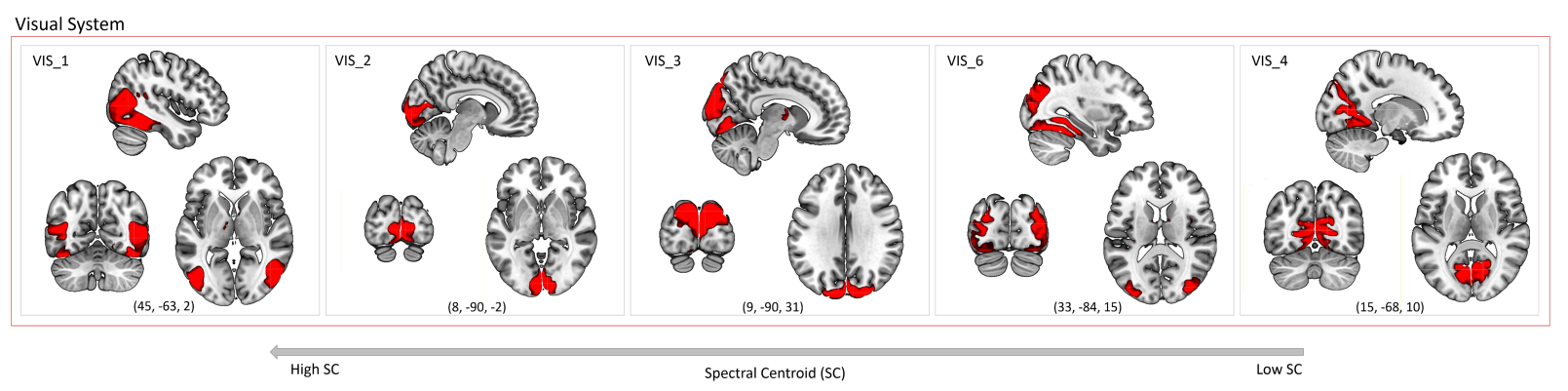

FIGURE 5 | Networks of the visual system ordered accordingly to their decreasing SC magnitude; displayed at the three most informative slices (MNI space).

When ordered accordingly to decreasing SC magnitude, the spatial distribution of brain areas constituting individual visual networks exhibits a rather characteristic pattern (see Figure 5). Networks encompassing predominantly early sensory areas in the striate cortex (primary visual area $\mathrm{V} 1$, calcarine sulcus) exhibit a higher SC (VIS_2, VIS_3), thus, their BOLD signal is strongly influenced by high-frequencies. Networks that encompass higher-order cortical areas along the visual stream, i.e., the extrastriate cortex (visual areas V2, V3, V4, and V5/MT) show comparably lower SC (VIS_6, VIS_4), thus, lower-frequencies dominate their BOLD signal. However, there is one exception; VIS_1 shows the highest SC among the visual networks, although it comprises higher-order visual area V5/MT in the middle temporal gyrus. This could be due to the extensive connections between area V5/MT and area V1 (Pascual-Leone and Walsh, 2001; Silvanto et al., 2005), and facilitate direct communication between these two visual areas. Importantly, the finding that networks comprising the same V5/MT area are located both at higher and lower end of the SC gradient could relate to multiple parallel processes being asynchronously undertaken by area V5/MT (for a review see Zeki, 2015). Overall, areas of the visual cortex are highly functionally specialized (Zeki et al., 1991); our results yield that different functional units within the visual system operate on distinct intrinsic dynamics regimescongruent with the sensorimotor-to-transmodal gradient of timescales.

Interestingly, the subsystems of the DMN (anterior and posterior) were localized at different ends of the power spectrum range, with the anterior and anterior-medial DMN showing relatively higher SC, and the posterior and posterior-lateral DMN showing relatively lower SC. This is indicative of differential contributions of spectral power to different subparts of the DMN, potentially reflecting different processes facilitated via different frequencies. The heterogeneity of the DMN architecture i.e., the subdivision into smaller anatomical-functional subsystems has been shown previously (Andrews-Hanna et al., 2010), and their interplay has been shown to be implicated in the psychopathology of various neuropsychiatric disorders including schizophrenia (Du et al., 2016), Alzheimer's disease (Damoiseaux et al., 2012), OCD (Beucke et al., 2014) and MDD (Zhu et al., 2012, 2017; Sambataro et al., 2014).
In summary, the grading of SC across RSNs points toward frequency-dependent functional specificity which is indicative of differentiated information integration processes being executed at different frequency scales, in different brain regions. It is in accordance with the results of Neufang et al. (2014) and Cocchi et al. (2016) which hint toward the frequency-driven directionality of information flow in the brain. Differences in spectra of BOLD signals across distinct brain networks have been reported before, via measures of scale-free properties (He et al., 2010; He, 2011). However, measures of scale-free properties alone are not well-suited for explaining findings of networkcharacteristic processes restricted to limited frequency bands. The SC, on the other hand, both reflects scale-free properties of BOLD signal and carries information about frequency-contentas is it affected by frequency-specific processes.

\section{Biological Relevance of Spectral Centroid Grading}

Power shifts of BOLD network fluctuations at rest within various frequency bands have been reported in several diseased states. A common tendency of increased power in high frequencies of the BOLD signal and decreased power in low frequencies has been observed in the ACC and insula (Malinen et al., 2010), as well as in the DMN (Baliki et al., 2011) in chronic pain patients; the same tendency was present across many RSNs-including the DMN-in bipolar disorder and schizophrenia (Garrity et al., 2007; Calhoun et al., 2011). The observations of our study are in line with these reports. The grading of SC of BOLD network fluctuations in MDD patients was found to deviate from the healthy orchestration pattern. Specifically, there was a shift of the $\mathrm{SC}$ of $\mathrm{SN}$ toward higher frequencies, which could be attributed to a relatively decreased power in low frequencies $(0.01-0.025 \mathrm{~Hz})$ and increased power in a broad range of higher frequencies $(0.15-0.25 \mathrm{~Hz})$ in patients, implying increased activity with characteristic times corresponding to this frequency range within the salience system.

Since the grading of the SC of BOLD network fluctuations is believed to be key to successful communication, we further investigated how shifts in BOLD fluctuations of the SN impact on its communication structure. A subsequent whole-brain seed-based FC analysis of SN enabled us to gain insight into the consequences of its altered dynamic and to specify brain regions toward which SN exhibited decreased FC in patients. 
In depression, a decreased FC of the SN toward the anterior DMN and one of the visual networks (VIS_4) was observed. Although there was no direct correlation between the SC of SN and depression severity scores, the SCcorr values of the networks to which SN connectivity was disrupted, exhibited a significant correlation with BDI scores. Inasmuch, we suspect an indirect influence that the shift in power spectrum of $\mathrm{SN}$ exerts on the severity of experienced symptoms. One possible mechanism underlying such a reaction chain could be that the decoupling of the SN from the anterior DMN and the visual network forces these networks to regain the lost oscillations synchronicity, and that such compensatory attempts would be mirrored through depression severity. We are, however, aware of the speculative character of this interpretation. Further investigations would be needed to support our point of view; this however, is beyond the scope of the current report.

\section{Grading of Activity Levels Across RSNs}

Different RSNs exhibit significantly distinct levels of BOLD activity, as quantified by PSC. Previously, both in the human and macaque cortex, widely distributed brain regions were found to show distinct ratios of excitatory and inhibitory receptor density (ExIn ratio) (van den Heuvel et al., 2016). In humans, regions displaying high ExIn ratios spanned across the precentral, superior frontal, orbitofrontal, supramarginal, superiotemporal, and angular gyri, and the IPL; while regions with relatively low ExIn ratios spanned across the parstriangularis, inferior frontal gyrus and the occipital lobe. Such distribution is to a high extent consistent with the spatial variation in network PSC observed in our study in Dataset 2, where the posteriorlateral DMN (main hubs in superiotemporal, supramarginal, and angular gyri) exhibited by far the highest PSC. It was followed by the sensorimotor network (SM_1; main hubs in the precentral and superior temporal gyri, IPL); the auditory network (main hubs in the superior- and middle temporal, and precentral gyri); and the anterior-medial DMN (main hubs in superior- and middle frontal gyri, and ACC). The lowest PSC was observed in one of the visual networks (VIS_5; main hubs in the lingual, fusiform and middle occipital gyri, and cuneus). We therefore speculate that network PSC is a measure which largely relates to the excitation loading and magnitude of neuronal activity within an ensemble of brain regions, and that the activity profile of a network can be broadly influenced by balanced impact of excitatory and inhibitory neurotransmitters and receptors.

However, in parallel to the coupling between neuronal activity and changes in absolute BOLD signal, it is important to note that differences in BOLD PSC may also be driven by differences in cerebral vascular reactivity (CVR) (Bandettini and Wong, 1997; Handwerker et al., 2007; Thomason et al., 2007), baseline venous oxygenation ( $\mathrm{Lu}$ et al., 2008), as well as baseline cerebral blood flow (CBF) (Liau and Liu, 2009).

\section{Biological Relevance of Activity Level Grading}

In addition to our finding of RSNs being characterized by specific PSC values, there was also a deviation in PSC values within networks spanning the occipital cortex in patient group.
Specifically, MDD patients showed significantly increased PSC in one of the visual networks (VIS_2; main hubs in the lingual gyrus, cuneus, fusiform gyrus, and occipital gyrus). Our observation of increased PSC in patients' visual networks can be interpreted in the context of Sanacora et al.'s (2004) reports where, relative to $\mathrm{HCs}, \mathrm{MDD}$ patients showed altered excitatory and inhibitory neurotransmitter levels in the occipital cortex. Precisely, patients exhibited reduced GABA and increased glutamate concentrations in the occipital cortex, along with decreased GABA/glutamate ratio. These findings, alongside with previous reports of $\mathrm{MDD}$ being associated with GABA dysfunction (Sanacora et al., 2000; Brambilla et al., 2003; Tunnicliff and Malatynska, 2003) as well as converging reports of normalization of occipital cortex GABA concentrations after targeted therapy (Sanacora et al., 2002, 2003), further support the notion that GABA function highly contributes to the pathophysiology and treatment of depression. Along the same lines, increased PSC of the visual networks, as revealed by our study, putatively reflects the twofold mechanism underlying the overall changes in cortical excitability: (1) deficits in inhibitory processes governed by reduced GABA concentration, accompanied by (2) excessive excitatory stimulation due to increased glutamate concentration.

Studies in FC analysis have established a link between activity of ventral tegmental area (VTA) of the SN with occipital regions. Seed-based analysis shows an anti-correlated relation between VTA and the visual areas (Tomasi and Volkow, 2014; Zhang et al., 2016). Interpreted as inhibitory influence of SN on these areas, SN malfunctioning could relate to less inhibition.

\section{LIMITATIONS}

In this study, we applied the SC as an informative summary measure reflecting spectral properties of RSNs. However, it is important to note that the SC is an averaged representative of the center of gravity of a network's full power spectrum over time. Recent studies have, however, shown that the frequency content of RSN fluctuations is dynamic in time (Yaesoubi et al., 2015, 2017). Another concern that could be expressed over our findings, is the undermined neural information content of high-frequency fMRI signal. It has been suggested that the higher band BOLD signal is primarily driven by confounding factors, such as physiological noise, and that information specific to RSNs is limited to lowest frequency range of 0.01$0.1 \mathrm{~Hz}$ (Cordes et al., 2001). However, there is convincing evidence that this assumption might be wrong, as studies report meaningful neural content and resting-state connectivity patterns at frequencies up to $0.25 \mathrm{~Hz}$ or even $0.75 \mathrm{~Hz}$ (Niazy et al., 2011; Boubela et al., 2013; Lewis et al., 2016). Furthermore, here we operate on ICA-derived time courses, which are likely to contain fewer artifacts than the raw fMRI time courses. Moreover, the caveat that relates to potential non-neural effects in the low-frequency fMRI signal $(<0.15 \mathrm{~Hz})$ should also be considered. In addition to scanner drifts, low frequencies can also reflect aliased high-frequency cardiac pulsations (Lowe et al., 1998; Bhattacharyya and Lowe, 2004) and slow physiological changes such as end-tidal $\mathrm{CO}_{2}$ fluctuations (Wise et al., 2004). 
The effects of cardiac pulsations on group differences in SC were minimal, since the pulsation rates did not differ between groups. However, $\mathrm{CO}_{2}$ effects can be quite strong and are unlikely to be separated by ICA, as they constitute a global confound distributed across all ICs in the brain.

\section{CONCLUSION}

In this work, we propose a new aggregate measure-the Spectral Centroid-which represents the "center of gravity" of the full power spectrum of individual RSN time courses. Based on a high-quality and large sample dataset of the Human Connectome Project, we show that there is a highly organized grading of SC across RSNs. This indicates a characteristic balance between specific frequencies involved in the power spectrum of each RSN. The occurrence of grading of SC across RSNs was replicated in an independent dataset, which further supports the validity of the proposed approach. Moreover, SC was shown to be a measure sensitive to power changes in BOLD network fluctuations in disease. In MDD, significantly increased SC was observed in the $\mathrm{SN}-\mathrm{a}$ system well-known to be implicated in depression. Following the preliminary indication of altered spectral properties of SN in depression, we selectively investigated the spectral power of BOLD network fluctuations within distinct frequency bands. Compared to HCs, increased contributions of high-frequencies and reduced contributions of low-frequencies to the BOLD signal of $\mathrm{SN}$ were revealed in depression. In summary, SC is a compact and reliable measure that allows to determine characteristics of power distribution of BOLD network fluctuations and spot key changes in disease.

\section{REFERENCES}

Allen, E. A., Erhardt, E. B., Damaraju, E., Gruner, W., Segall, J. M., Silva, R. F., et al. (2011). A baseline for the multivariate comparison of resting-state networks. Front. Syst. Neurosci. 5:2. doi: 10.3389/fnsys.2011.00002

American Psychiatric Association (2000). Diagnostic and Statistical Manual of Mental Disorders: DSM-IV-TR. Washington, DC: American Psychiatric Association.

Andrews-Hanna, J. R., Reidler, J. S., Sepulcre, J., Poulin, R., and Buckner, R. L. (2010). Functional-anatomic fractionation of the brain's default network. Neuron 65, 550-562. doi: 10.1016/j.neuron.2010.02.005

Badhwar, A., Tam, A., Dansereau, C., Orban, P., Hoffstaedter, F., and Bellec, P. (2017). Resting-state network dysfunction in Alzheimer's disease: a systematic review and meta-analysis. Alzheimers Dement. 8, 73-85. doi: 10.1016/J.DADM. 2017.03.007

Baldassano, C., Chen, J., Zadbood, A., Pillow, J. W., Hasson, U., and Norman, K. A. (2017). Discovering event structure in continuous narrative perception and memory. Neuron 95, 709-721.e5. doi: 10.1016/j.neuron.2017. 06.041

Baliki, M. N., Baria, A. T., and Apkarian, A. V. (2011). The cortical rhythms of chronic back pain. J. Neurosci. 31, 13981-13990. doi: 10.1523/JNEUROSCI. 1984-11.2011

Bandettini, P. A., and Wong, E. C. (1997). A hypercapnia-based normalization method for improved spatial localization of human brain activation with fMRI. NMR Biomed. 10, 197-203. doi: 10.1002/(SICI)1099-1492(199706/08)10: 4/5<197::AID-NBM466>3.0.CO;2-S

\section{ETHICS STATEMENT}

This study was carried out in accordance with the recommendations of Human Research Committee guidelines of the Klinikum rechts der Isar, Technische Universität München with written informed consent from all subjects. All subjects gave written informed consent in accordance with the Declaration of Helsinki. The protocol was approved by the Human Research Committee of the Klinikum rechts der Isar, Technische Universität München.

\section{AUTHOR CONTRIBUTIONS}

$\mathrm{AR}, \mathrm{CS}$, and AW conceived the research. AR and CM preprocessed the data. AR and AW analyzed the data and prepared the figures. AR, CC, CS, and AW interpreted the results. AR drafted the manuscript. AR, CC, CM, CS, and AW edited and revised the manuscript. AR, CC, SG, CM, CS, and AW approved the final version of the manuscript.

\section{FUNDING}

AR was supported by the Studienstiftung des deutschen Volkes.

\section{SUPPLEMENTARY MATERIAL}

The Supplementary Material for this article can be found online at: https://www.frontiersin.org/articles/10.3389/fnhum. 2018.00436/full\#supplementary-material

Baria, A. T., Baliki, M. N., Parrish, T., and Apkarian, A. V. (2011). Anatomical and functional assemblies of brain BOLD oscillations. J. Neurosci. 31, 7910-7919. doi: 10.1523/JNEUROSCI.1296-11.2011

Beall, E. B., and Lowe, M. J. (2007). Isolating physiologic noise sources with independently determined spatial measures. Neuroimage 37, 1286-1300. doi: 10.1016/j.neuroimage.2007.07.004

Beck, A. T., Ward, C. H., Mendelson, M., Mock, J., and Erbaugh, J. (1961). An inventory for measuring depression. Arch. Gen. Psychiatry 4, 561-571. doi: 10.1001/archpsyc.1961.01710120031004

Beucke, J. C., Sepulcre, J., Eldaief, M. C., Sebold, M., Kathmann, N., and Kaufmann, C. (2014). Default mode network subsystem alterations in obsessive-compulsive disorder. Br. J. Psychiatry 205, 376-382. doi: 10.1192/bjp. bp.113.137380

Bhattacharyya, P. K., and Lowe, M. J. (2004). Cardiac-induced physiologic noise in tissue is a direct observation of cardiac-induced fluctuations. Magn. Reson. Imaging 22, 9-13. doi: 10.1016/j.mri.2003.08.003

Biswal, B., Yetkin, F. Z., Haughton, V. M., and Hyde, J. S. (1995). Functional connectivity in the motor cortex of resting human brain using echo-planar MRI. Magn. Reson. Med. 34, 537-541. doi: 10.1002/mrm.1910340409

Boubela, R. N., Kalcher, K., Huf, W., Kronnerwetter, C., Filzmoser, P., and Moser, E. (2013). Beyond noise: using temporal ICA to extract meaningful information from high-frequency FMRI signal fluctuations during rest. Front. Hum. Neurosci. 7:168. doi: 10.3389/fnhum.2013.00168

Brambilla, P., Perez, J., Barale, F., Schettini, G., and Soares, J. C. (2003). GABAergic dysfunction in mood disorders. Mol. Psychiatry 8, 721-737. doi: 10.1038/sj.mp. 4001362 
Buckner, R. L., Andrews-Hanna, J. R., and Schacter, D. L. (2008). The brain's default network. Ann. N. Y. Acad. Sci. 1124, 1-38. doi: 10.1196/annals.1440.011

Buzsáki, G. (2006). Rhythms of the Brain. Oxford: Oxford University Press. doi: 10.1093/acprof:oso/9780195301069.001.0001

Calhoun, V. D., Adali, T., Pearlson, G. D., and Pekar, J. J. (2001). Spatial and temporal independent component analysis of functional MRI data containing a pair of task-related waveforms. Hum. Brain Mapp. 13, 43-53. doi: 10.1002/hbm. 1024

Calhoun, V. D., Sui, J., Kiehl, K., Turner, J., Allen, E., and Pearlson, G. (2011). Exploring the psychosis functional connectome: aberrant intrinsic networks in schizophrenia and bipolar disorder. Front. Psychiatry 2:75. doi: 10.3389/fpsyt. 2011.00075

Chaudhuri, R., Knoblauch, K., Gariel, M.-A., Kennedy, H., and Wang, X.-J. (2015). A large-scale circuit mechanism for hierarchical dynamical processing in the primate cortex. Neuron 88, 419-431. doi: 10.1016/j.neuron.2015.09.008

Cocchi, L., Sale, M. V., L Gollo, L., Bell, P. T., Nguyen, V. T., Zalesky, A., et al. (2016). A hierarchy of timescales explains distinct effects of local inhibition of primary visual cortex and frontal eye fields. eLife 5:e15252. doi: 10.7554/eLife. 15252

Cordes, D., Haughton, V. M., Arfanakis, K., Carew, J. D., Turski, P. A., Moritz, C. H., et al. (2001). Frequencies contributing to functional connectivity in the cerebral cortex in "resting-state" data. Am. J. Neuroradiol. 22, 1326-1333.

Damoiseaux, J. S., Prater, K. E., Miller, B. L., and Greicius, M. D. (2012). Functional connectivity tracks clinical deterioration in Alzheimer's disease. Neurobiol. Aging 33, 828.e19-828.e30. doi: 10.1016/j.neurobiolaging.2011.06.024

Ding, N., Melloni, L., Zhang, H., Tian, X., and Poeppel, D. (2016). Cortical tracking of hierarchical linguistic structures in connected speech. Nat. Neurosci. 19, 158-164. doi: 10.1038/nn.4186

Dong, D., Wang, Y., Chang, X., Luo, C., and Yao, D. (2018). Dysfunction of large-scale brain networks in schizophrenia: a meta-analysis of resting-state functional connectivity. Schizophr. Bull. 44, 168-181. doi: 10.1093/schbul/ sbx034

Du, Y., Pearlson, G. D., Yu, Q., He, H., Lin, D., Sui, J., et al. (2016). Interaction among subsystems within default mode network diminished in schizophrenia patients: a dynamic connectivity approach. Schizophr. Res. 170, 55-65. doi: 10.1016/j.schres.2015.11.021

First, M. B., Spitzer, R. L., Gibbon, M., and Williams, J. B. W. (1996). Structured Clinical Interview for DSM-IV Axis I Disorders, Clinician Version (SCDIV-CV). Washington, DC: American Psychiatric Press, Inc.

Fox, M. D., and Raichle, M. E. (2007). Spontaneous fluctuations in brain activity observed with functional magnetic resonance imaging. Nat. Rev. Neurosci. 8, 700-711. doi: 10.1038/nrn2201

Fox, M. D., Snyder, A. Z., Vincent, J. L., Corbetta, M., Van Essen, D. C., and Raichle, M. E. (2005). The human brain is intrinsically organized into dynamic, anticorrelated functional networks. Proc. Natl. Acad. Sci. U.S.A. 102, 9673-9678. doi: $10.1073 /$ pnas. 0504136102

Garrity, A. G., Pearlson, G. D., McKiernan, K., Lloyd, D., Kiehl, K. A., and Calhoun, V. D. (2007). Aberrant "default mode" functional connectivity in schizophrenia. Am. J. Psychiatry 164, 450-457. doi: 10.1176/ajp.2007.164.3.450

Gläscher, J. (2009). Visualization of group inference data in functional neuroimaging. Neuroinformatics 7, 73-82. doi: 10.1007/s12021-008-9042-x

Glasser, M. F., Sotiropoulos, S. N., Wilson, J. A., Coalson, T. S., Fischl, B., Andersson, J. L., et al. (2013). The minimal preprocessing pipelines for the Human Connectome Project. Neuroimage 80, 105-124. doi: 10.1016/j. neuroimage.2013.04.127

Gohel, S. R., and Biswal, B. B. (2015). Functional integration between brain regions at rest occurs in multiple-frequency bands. Brain Connect. 5, 23-34. doi: 10. 1089/brain.2013.0210

Gollo, L. L., Roberts, J. A., and Cocchi, L. (2017). Mapping how local perturbations influence systems-level brain dynamics. Neuroimage 160, 97-112. doi: 10.1016/ J.NEUROIMAGE.2017.01.057

Gollo, L. L., Zalesky, A., Hutchison, R. M., van den Heuvel, M., and Breakspear, M. (2015). Dwelling quietly in the rich club: brain network determinants of slow cortical fluctuations. Philos. Trans. R. Soc. Lond. B Biol. Sci. 370:20140165. doi: $10.1098 /$ rstb.2014.0165

Greicius, M. D., Flores, B. H., Menon, V., Glover, G. H., Solvason, H. B., Kenna, H., et al. (2007). Resting-state functional connectivity in major depression: abnormally increased contributions from subgenual cingulate cortex and thalamus. Biol. Psychiatry 62, 429-437. doi: 10.1016/j.biopsych.2006.09.020

Greicius, M. D., Krasnow, B., Reiss, A. L., and Menon, V. (2003). Functional connectivity in the resting brain: a network analysis of the default mode hypothesis. Proc. Natl. Acad. Sci. U.S.A. 100, 253-258. doi: 10.1073/pnas. 0135058100

Greicius, M. D., Srivastava, G., Reiss, A. L., and Menon, V. (2004). Defaultmode network activity distinguishes Alzheimer's disease from healthy aging: evidence from functional MRI. Proc. Natl. Acad. Sci. U.S.A. 101, 4637-4642. doi: $10.1073 /$ pnas.0308627101

Griffanti, L., Salimi-Khorshidi, G., Beckmann, C. F., Auerbach, E. J., Douaud, G. Sexton, C. E., et al. (2014). ICA-based artefact removal and accelerated fMRI acquisition for improved resting state network imaging. Neuroimage 95, 232247. doi: 10.1016/j.neuroimage.2014.03.034

Gürsel, D. A., Avram, M., Sorg, C., Brandl, F., and Koch, K. (2018). Frontoparietal areas link impairments of large-scale intrinsic brain networks with aberrant fronto-striatal interactions in OCD: a meta-analysis of restingstate functional connectivity. Neurosci. Biobehav. Rev. 87, 151-160. doi: 10 1016/J.NEUBIOREV.2018.01.016

Hamilton, M. (1960). A rating scale for depression. J. Neurol. Neurosurg. Psychiatry 23, 56-62. doi: 10.1136/jnnp.23.1.56

Handwerker, D. A., Gazzaley, A., Inglis, B. A., and D’Esposito, M. (2007). Reducing vascular variability of fMRI data across aging populations using a breathholding task. Hum. Brain Mapp. 28, 846-859. doi: 10.1002/hbm.20307

Hasson, U., Chen, J., and Honey, C. J. (2015). Hierarchical process memory: memory as an integral component of information processing. Trends Cogn. Sci. 19, 304-313. doi: 10.1016/j.tics.2015.04.006

Hasson, U., Yang, E., Vallines, I., Heeger, D. J., and Rubin, N. (2008). A hierarchy of temporal receptive windows in human cortex. J. Neurosci. 28, 2539-2550. doi: 10.1523/JNEUROSCI.5487-07.2008

He, B. J. (2011). Scale-free properties of the functional magnetic resonance imaging signal during rest and task. J. Neurosci. 31, 13786-13795. doi: 10.1523/ JNEUROSCI.2111-11.2011

He, B. J., Zempel, J. M., Snyder, A. Z., and Raichle, M. E. (2010). The temporal structures and functional significance of scale-free brain activity. Neuron 66, 353-369. doi: 10.1016/j.neuron.2010.04.020

Honey, C. J., Thesen, T., Donner, T. H., Silbert, L. J., Carlson, C. E., Devinsky, O., et al. (2012). Slow cortical dynamics and the accumulation of information over long timescales. Neuron 76, 423-434. doi: 10.1016/j.neuron.2012. 08.011

Huang, Z., Liu, X., Mashour, G. A., and Hudetz, A. G. (2018). Timescales of intrinsic BOLD signal dynamics and functional connectivity in pharmacologic and neuropathologic states of unconsciousness. J. Neurosci. 38, 2304-2317. doi: 10.1523/JNEUROSCI.2545-17.2018

Huntenburg, J. M., Bazin, P.-L., Goulas, A., Tardif, C. L., Villringer, A., and Margulies, D. S. (2017). A systematic relationship between functional connectivity and intracortical myelin in the human cerebral cortex. Cereb. Cortex 27, 981-997. doi: 10.1093/cercor/bhx030

Huntenburg, J. M., Bazin, P.-L., and Margulies, D. S. (2018). Large-scale gradients in human cortical organization. Trends Cogn. Sci. 22, 21-31. doi: 10.1016/j.tics. 2017.11.002

Huth, A. G., de Heer, W. A., Griffiths, T. L., Theunissen, F. E., and Gallant, J. L. (2016). Natural speech reveals the semantic maps that tile human cerebral cortex. Nature 532, 453-458. doi: 10.1038/nature 17637

Kaiser, R. H., Andrews-Hanna, J. R., Wager, T. D., and Pizzagalli, D. A. (2015). Large-scale network dysfunction in major depressive disorder: a meta-analysis of resting-state functional connectivity. JAMA Psychiatry 72, 603-611. doi: 10. 1001/jamapsychiatry.2015.0071

Kalcher, K., Boubela, R. N., Huf, W., Bartova, L., Kronnerwetter, C., Derntl, B., et al. (2014). The spectral diversity of resting-state fluctuations in the human brain. PLoS One 9:e93375. doi: 10.1371/journal.pone.0093375

Lerner, Y., Honey, C. J., Silbert, L. J., and Hasson, U. (2011). Topographic mapping of a hierarchy of temporal receptive windows using a narrated story. J. Neurosci. 31, 2906-2915. doi: 10.1523/JNEUROSCI.3684-10.2011

Lewis, L. D., Setsompop, K., Rosen, B. R., and Polimeni, J. R. (2016). Fast fMRI can detect oscillatory neural activity in humans. Proc. Natl. Acad. Sci. U.S.A. 113, E6679-E6685. doi: 10.1073/pnas.1608117113 
Liau, J., and Liu, T. T. (2009). Inter-subject variability in hypercapnic normalization of the BOLD fMRI response. Neuroimage 45, 420-430. doi: 10.1016/j. neuroimage.2008.11.032

Lowe, M. J., Mock, B. J., and Sorenson, J. A. (1998). Functional connectivity in single and multislice echoplanar imaging using resting-state fluctuations. Neuroimage 7, 119-132. doi: 10.1006/nimg.1997.0315

Lu, H., Zhao, C., Ge, Y., and Lewis-Amezcua, K. (2008). Baseline blood oxygenation modulates response amplitude: physiologic basis for intersubject variations in functional MRI signals. Magn. Reson. Med. 60, 364-372. doi: 10.1002/mrm. 21686

Malinen, S., Vartiainen, N., Hlushchuk, Y., Koskinen, M., Ramkumar, P., Forss, N., et al. (2010). Aberrant temporal and spatial brain activity during rest in patients with chronic pain. Proc. Natl. Acad. Sci. U.S.A. 107, 6493-6497. doi: 10.1073/ pnas. 1001504107

Manoliu, A., Riedl, V., Doll, A., Bäuml, J. G., Mühlau, M., Schwerthöffer, D., et al. (2013). Insular dysfunction reflects altered between-network connectivity and severity of negative symptoms in schizophrenia during psychotic remission. Front. Hum. Neurosci. 7:216. doi: 10.3389/fnhum.2013.00216

Margulies, D. S., Ghosh, S. S., Goulas, A., Falkiewicz, M., Huntenburg, J. M., Langs, G., et al. (2016). Situating the default-mode network along a principal gradient of macroscale cortical organization. Proc. Natl. Acad. Sci. U.S.A. 113, 12574-12579. doi: 10.1073/pnas.1608282113

Meng, C., Brandl, F., Tahmasian, M., Shao, J., Manoliu, A., Scherr, M., et al. (2014). Aberrant topology of striatum's connectivity is associated with the number of episodes in depression. Brain 137, 598-609. doi: 10.1093/brain/awt290

Menon, V., and Uddin, L. Q. (2010). Saliency, switching, attention and control: a network model of insula function. Brain Struct. Funct. 214, 655-667. doi: 10.1007/s00429-010-0262-0

Murphy, K., Bodurka, J., and Bandettini, P. A. (2007). How long to scan? The relationship between fMRI temporal signal to noise ratio and necessary scan duration. Neuroimage 34, 565-574. doi: 10.1016/j.neuroimage.2006.09.032

Murray, J. D., Bernacchia, A., Freedman, D. J., Romo, R., Wallis, J. D., Cai, X., et al. (2014). A hierarchy of intrinsic timescales across primate cortex. Nat. Neurosci. 17, 1661-1663. doi: 10.1038/nn.3862

Neufang, S., Akhrif, A., Riedl, V., Förstl, H., Kurz, A., Zimmer, C., et al. (2014). Predicting effective connectivity from resting-state networks in healthy elderly and patients with prodromal Alzheimer's disease. Hum. Brain Mapp. 35, 954963. doi: $10.1002 / \mathrm{hbm} .22226$

Niazy, R. K., Xie, J., Miller, K., Beckmann, C. F., and Smith, S. M. (2011). Spectral characteristics of resting state networks. Prog. Brain Res. 193, 259-276. doi: 10.1016/B978-0-444-53839-0.00017-X

Pascual-Leone, A., and Walsh, V. (2001). Fast backprojections from the motion to the primary visual area necessary for visual awareness. Science 292, 510-512. doi: 10.1126/science.1057099

Poldrack, R. A., Baker, C. I., Durnez, J., Gorgolewski, K. J., Matthews, P. M., Munafò, M. R., et al. (2017). Scanning the horizon: towards transparent and reproducible neuroimaging research. Nat. Rev. Neurosci. 18, 115-126. doi: 10. 1038/nrn.2016.167

Raichle, M. E., MacLeod, A. M., Snyder, A. Z., Powers, W. J., Gusnard, D. A., and Shulman, G. L. (2001). A default mode of brain function. Proc. Natl. Acad. Sci. U.S.A. 98, 676-682. doi: 10.1073/pnas.98.2.676

Rotarska-Jagiela, A., van de Ven, V., Oertel-Knöchel, V., Uhlhaas, P. J., Vogeley, K., and Linden, D. E. J. (2010). Resting-state functional network correlates of psychotic symptoms in schizophrenia. Schizophr. Res. 117, 21-30. doi: 10.1016/ j.schres.2010.01.001

Salimi-Khorshidi, G., Douaud, G., Beckmann, C. F., Glasser, M. F., Griffanti, L., and Smith, S. M. (2014). Automatic denoising of functional MRI data: combining independent component analysis and hierarchical fusion of classifiers. Neuroimage 90, 449-468. doi: 10.1016/j.neuroimage.2013. 11.046

Salvador, R., Martínez, A., Pomarol-Clotet, E., Gomar, J., Vila, F., Sarró, S., et al. (2008). A simple view of the brain through a frequency-specific functional connectivity measure. Neuroimage 39, 279-289. doi: 10.1016/j.neuroimage. 2007.08.018

Sambataro, F., Wolf, N. D., Pennuto, M., Vasic, N., and Wolf, R. C. (2014). Revisiting default mode network function in major depression: evidence for disrupted subsystem connectivity. Psychol. Med. 44, 2041-2051. doi: 10.1017/ S0033291713002596
Sanacora, G., Gueorguieva, R., Epperson, C. N., Wu, Y.-T., Appel, M., Rothman, D. L., et al. (2004). Subtype-specific alterations of $\gamma$-aminobutyric acid and glutamatein patients with major depression. Arch. Gen. Psychiatry 61, 705-713. doi: 10.1001/archpsyc.61.7.705

Sanacora, G., Mason, G. F., and Krystal, J. H. (2000). Impairment of GABAergic transmission in depression: new insights from neuroimaging studies. Crit. Rev. Neurobiol. 14, 23-45. doi: 10.1615/CritRevNeurobiol.v14.i1.20

Sanacora, G., Mason, G. F., Rothman, D. L., Hyder, F., Ciarcia, J. J., Ostroff, R. B., et al. (2003). Increased cortical GABA concentrations in depressed patients receiving ECT. Am. J. Psychiatry 160, 577-579. doi: 10.1176/appi.ajp.160.3.577

Sanacora, G., Mason, G. F., Rothman, D. L., and Krystal, J. H. (2002). Increased occipital cortex GABA concentrations in depressed patients after therapy with selective serotonin reuptake inhibitors. Am. J. Psychiatry 159, 663-665. doi: 10.1176/appi.ajp.159.4.663

Sasai, S., Homae, F., Watanabe, H., Sasaki, A. T., Tanabe, H. C., Sadato, N., et al. (2014). Frequency-specific network topologies in the resting human brain. Front. Hum. Neurosci. 8:1022. doi: 10.3389/fnhum.2014.01022

Seeley, W. W., Menon, V., Schatzberg, A. F., Keller, J., Glover, G. H., Kenna, H., et al. (2007). Dissociable intrinsic connectivity networks for salience processing and executive control. J. Neurosci. 27, 2349-2356. doi: 10.1523/JNEUROSCI. 5587-06.2007

Sheline, Y. I., and Raichle, M. E. (2013). Resting state functional connectivity in preclinical Alzheimer's disease. Biol. Psychiatry 74, 340-347. doi: 10.1016/j. biopsych.2012.11.028

Silvanto, J., Cowey, A., Lavie, N., and Walsh, V. (2005). Striate cortex (V1) activity gates awareness of motion. Nat. Neurosci. 8, 143-144. doi: 10.1038/nn1379

Smith, S. M., Beckmann, C. F., Andersson, J., Auerbach, E. J., Bijsterbosch, J., Douaud, G., et al. (2013). Resting-state fMRI in the human connectome project. Neuroimage 80, 144-168. doi: 10.1016/j.neuroimage.2013.05.039

Sorg, C., Riedl, V., Muhlau, M., Calhoun, V. D., Eichele, T., Laer, L., et al. (2007). Selective changes of resting-state networks in individuals at risk for Alzheimer's disease. Proc. Natl. Acad. Sci. U.S.A. 104, 18760-18765. doi: 10.1073/pnas. 0708803104

Spitzer, R. L., Williams, J. B., Gibbon, M., and First, M. B. (1992). The structured clinical interview for DSM-III-R (SCID). I: history, rationale, and description. Arch. Gen. Psychiatry 49, 624-629. doi: 10.1001/archpsyc.1992.01820080032005

Stephens, G. J., Honey, C. J., and Hasson, U. (2013). A place for time: the spatiotemporal structure of neural dynamics during natural audition. J. Neurophysiol. 110, 2019-2026. doi: 10.1152/jn.00268.2013

Thomason, M. E., Foland, L. C., and Glover, G. H. (2007). Calibration of BOLD fMRI using breath holding reduces group variance during a cognitive task. Hum. Brain Mapp. 28, 59-68. doi: 10.1002/hbm.20241

Thompson, W. H., and Fransson, P. (2015). The frequency dimension of fMRI dynamic connectivity: network connectivity, functional hubs and integration in the resting brain. Neuroimage 121, 227-242. doi: 10.1016/j.neuroimage.2015. 07.022

Tomasi, D., and Volkow, N. D. (2014). Functional connectivity of Substantia nigra and ventral tegmental area: maturation during adolescence and effects of ADHD. Cereb. Cortex 24, 935-944. doi: 10.1093/cercor/bhs382

Tunnicliff, G., and Malatynska, E. (2003). Central GABAergic systems and depressive Illness. Neurochem. Res. 28, 965-976. doi: 10.1023/A:1023287729363

Uğurbil, K., Xu, J., Auerbach, E. J., Moeller, S., Vu, A. T., Duarte-Carvajalino, J. M., et al. (2013). Pushing spatial and temporal resolution for functional and diffusion MRI in the Human Connectome Project. Neuroimage 80, 80-104. doi: 10.1016/J.NEUROIMAGE.2013.05.012

van den Heuvel, M. P., Scholtens, L. H., Turk, E., Mantini, D., Vanduffel, W., and Feldman Barrett, L. (2016). Multimodal analysis of cortical chemoarchitecture and macroscale fMRI resting-state functional connectivity. Hum. Brain Mapp. 37, 3103-3113. doi: 10.1002/hbm.23229

Van Dijk, K. R. A., Sabuncu, M. R., and Buckner, R. L. (2012). The influence of head motion on intrinsic functional connectivity MRI. Neuroimage 59, 431-438. doi: 10.1016/j.neuroimage.2011.07.044

Van Essen, D. C., Smith, S. M., Barch, D. M., Behrens, T. E. J., Yacoub, E., Ugurbil, K., et al. (2013). The WU-Minn human connectome project: an overview. Neuroimage 80, 62-79. doi: 10.1016/j.neuroimage.2013.05.041

Vincent, J. L., Kahn, I., Snyder, A. Z., Raichle, M. E., and Buckner, R. L. (2008). Evidence for a frontoparietal control system revealed by intrinsic functional connectivity. J. Neurophysiol. 100, 3328-3342. doi: 10.1152/jn.90355.2008 
Wang, K., Liang, M., Wang, L., Tian, L., Zhang, X., Li, K., et al. (2007). Altered functional connectivity in early Alzheimer's disease: a restingstate fMRI study. Hum. Brain Mapp. 28, 967-978. doi: 10.1002/hbm. 20324

Wang, L., Hermens, D. F., Hickie, I. B., and Lagopoulos, J. (2012). A systematic review of resting-state functional-MRI studies in major depression. J. Affect. Disord. 142, 6-12. doi: 10.1016/J.JAD.2012.04.013

Wise, R. G., Ide, K., Poulin, M. J., and Tracey, I. (2004). Resting fluctuations in arterial carbon dioxide induce significant low frequency variations in BOLD signal. Neuroimage 21, 1652-1664. doi: 10.1016/j.neuroimage.2003.11.025

Wu, C. W., Chen, C.-L., Liu, P.-Y., Chao, Y.-P., Biswal, B. B., and Lin, C.-P. (2011). Empirical evaluations of slice-timing, smoothing, and normalization effects in seed-based, resting-state functional magnetic resonance imaging analyses. Brain Connect. 1, 401-410. doi: 10.1089/brain.2011.0018

Wu, C. W., Gu, H., Lu, H., Stein, E. A., Chen, J.-H., and Yang, Y. (2008). Frequency specificity of functional connectivity in brain networks. Neuroimage 42, 1047-1055. doi: 10.1016/j.neuroimage.2008.05.035

Yaesoubi, M., Allen, E. A., Miller, R. L., and Calhoun, V. D. (2015). Dynamic coherence analysis of resting fMRI data to jointly capture state-based phase, frequency, and time-domain information. Neuroimage 120, 133-142. doi: 10. 1016/j.neuroimage.2015.07.002

Yaesoubi, M., Miller, R. L., Calhoun, V. D., Cetin, M., Miller, R., and Pearlson, G. (2017). Time-varying spectral power of resting-state fMRI networks reveal cross-frequency dependence in dynamic connectivity. PLoS One 12:e171647. doi: 10.1371 /journal.pone.0171647

Zeki, S. (2015). Area V5-a microcosm of the visual brain. Front. Integr. Neurosci. 9:21. doi: 10.3389/fnint.2015.00021

Zeki, S., Watson, J. D., Lueck, C. J., Friston, K. J., Kennard, C., and Frackowiak, R. S. (1991). A direct demonstration of functional specialization in human visual cortex. J. Neurosci. 11, 641-649. doi: 10.1523/JNEUROSCI.11-03-00641. 1991

Zhang, S., Hu, S., Chao, H. H., and Li, C.-S. R. (2016). Resting-state functional connectivity of the locus coeruleus in humans: in comparison with the ventral tegmental area/Substantia Nigra pars compacta and the effects of age. Cereb. Cortex 26, 3413-3427. doi: 10.1093/cercor/bhv172

Zhong, X., Pu, W., and Yao, S. (2016). Functional alterations of fronto-limbic circuit and default mode network systems in first-episode, drug-naïve patients with major depressive disorder: a meta-analysis of resting-state fMRI data. J. Affect. Disord. 206, 280-286. doi: 10.1016/J.JAD.2016.09.005

Zhu, X., Wang, X., Xiao, J., Liao, J., Zhong, M., Wang, W., et al. (2012). Evidence of a dissociation pattern in resting-state default mode network connectivity in first-episode, treatment-naive major depression patients. Biol. Psychiatry 71, 611-617. doi: 10.1016/J.BIOPSYCH.2011.10.035

Zhu, X., Zhu, Q., Shen, H., Liao, W., and Yuan, F. (2017). Rumination and default mode network subsystems connectivity in first-episode, drug-naive young patients with major depressive disorder. Sci. Rep. 7:43105. doi: 10.1038/ srep43105

Zhu, Y., Fan, Q., Zhang, H., Qiu, J., Tan, L., Xiao, Z., et al. (2016). Altered intrinsic insular activity predicts symptom severity in unmedicated obsessivecompulsive disorder patients: a resting state functional magnetic resonance imaging study. BMC Psychiatry 16:104. doi: 10.1186/s12888-016-0806-9

Zuo, X. N., Di Martino, A., Kelly, C., Shehzad, Z. E., Gee, D. G., Klein, D. F., et al. (2010). The oscillating brain: complex and reliable. Neuroimage 49, 1432-1445. doi: 10.1016/j.neuroimage.2009.09.037

Conflict of Interest Statement: The authors declare that the research was conducted in the absence of any commercial or financial relationships that could be construed as a potential conflict of interest.

Copyright (C) 2018 Ries, Chang, Glim, Meng, Sorg and Wohlschläger. This is an open-access article distributed under the terms of the Creative Commons Attribution License (CC BY). The use, distribution or reproduction in other forums is permitted, provided the original author(s) and the copyright owner(s) are credited and that the original publication in this journal is cited, in accordance with accepted academic practice. No use, distribution or reproduction is permitted which does not comply with these terms. 